\title{
Tensorial generalization of characters
}

\author{
H. Itoyama, ${ }^{a}$ A. Mironov ${ }^{b, c, d}$ and A. Morozov ${ }^{e, c, d}$ \\ ${ }^{a}$ Nambu Yoichiro Institute of Theoretical and Experimental Physics (NITEP) and \\ Department of Mathematics and Physics, Graduate School of Science, Osaka City University, \\ 3-3-138, Sugimoto, Sumiyoshi-ku, Osaka, 558-8585, Japan \\ ${ }^{b}$ I.E.Tamm Theory Department, Lebedev Physics Institute, \\ Leninsky prospect, 53, Moscow 119991, Russia \\ ${ }^{c}$ Institute for Theoretical and Experimental Physics (ITEP), \\ B. Cheremushkinskaya, 25, Moscow, 117259, Russia \\ ${ }^{d}$ Institute for Information Transmission Problems, \\ Bolshoy Karetny per. 19, build.1, Moscow 127051 Russia \\ ${ }^{e}$ Moscow Institute of Physics and Technology (MIPT), \\ Dolgoprudny, 141701, Russia \\ E-mail: itoyama@sci.osaka-cu.ac.jp, mironov@lpi.ru, morozov@itep.ru
}

ABSTRACT: In rainbow tensor models, which generalize rectangular complex matrix model $(\mathrm{RCM})$ and possess a huge gauge symmetry $\mathrm{U}\left(N_{1}\right) \times \ldots \times \mathrm{U}\left(N_{r}\right)$, we introduce a new sub-basis in the linear space of gauge invariant operators, which is a redundant basis in the space of operators with non-zero Gaussian averages. Its elements are labeled by $r$-tuples of Young diagrams of a given size equal to the power of tensor field. Their tensor model averages are just products of dimensions: $\left\langle\chi_{R_{1}, \ldots, R_{r}}\right\rangle \sim C_{R_{1}, \ldots, R_{r}} D_{R_{1}}\left(N_{1}\right) \ldots D_{R_{r}}\left(N_{r}\right)$ of representations $R_{i}$ of the linear group $\mathrm{SL}\left(N_{i}\right)$, with $C_{R_{1}, \ldots, R_{r}}$ made of the ClebschGordan coefficients of representations $R_{i}$ of the symmetric group. Moreover, not only the averages, but the operators $\chi_{\vec{R}}$ themselves exist only when these $C_{\vec{R}}$ are non-vanishing. This sub-basis is much similar to the basis of characters (Schur functions) in matrix models, which is distinguished by the property 〈character〉 $\sim$ character, which opens a way to lift the notion and the theory of characters (Schur functions) from matrices to tensors. In particular, operators $\chi_{\vec{R}}$ are eigenfunctions of operators which generalize the usual cut-andjoin operators $\hat{W}$; they satisfy orthogonality conditions similar to the standard characters, but they do not form a full linear basis for all gauge-invariant operators, only for those which have non-vanishing Gaussian averages.

Keywords: Matrix Models, Random Systems

ArXiv EPrint: 1909.06921 


\section{Contents}

1 Introduction $\quad 2$

2 The main idea 5

2.1 Operators with a given symmetry 5

2.2 RCM 6

$\begin{array}{lll}2.3 & \text { Aristotelian model } & 7\end{array}$

3 Generalized characters $\quad 10$

3.1 Some basic properties of symmetric group characters 10

3.2 RCM, $r=2 \quad 11$

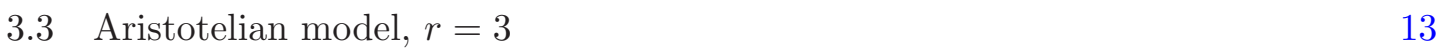

$\begin{array}{ll}3.4 & \text { Vanishing of } \chi_{\vec{R}} \text { with forbidden symmetries } \\ \end{array}$

3.5 Generalized characters as a basis for operators with non-vanishing Gaussian averages 14

$\begin{array}{lll}3.6 & \text { Background metrics } & 15\end{array}$

$\begin{array}{lll}3.7 & \text { Other rainbow models, arbitrary } r & 15\end{array}$

4 Orthogonality of generalized characters $\quad 16$

$\begin{array}{ll}4.1 \text { The problem } & 16\end{array}$

$\begin{array}{ll}\text { 4.2 RCM, } r=2 \text {, through ordinary characters } & 16\end{array}$

$\begin{array}{lll}4.3 & \text { RCM, } r=2 \text {, through bi-characters } & 17\end{array}$

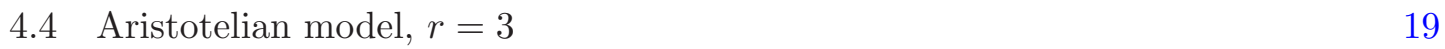

4.5 Other rainbow models, arbitrary $r \quad 20$

4.6 Generalized Cauchy formula 20

$5 \hat{W}$-operators $\quad \mathbf{2 1}$

5.1 Cut-and-join operators of $[52,53] 21$

$5.2 \hat{W}$-operators in rainbow tensor models 22

$\begin{array}{lll}5.3 & \mathrm{RCM}, r=2 & 23\end{array}$

5.4 Aristotelian model, $r=3 \quad 24$

5.5 $\hat{W}$-operators in $\xi$-variables 24

$\begin{array}{ll}\text { 5.5.1 The case of RCM, } r=2 & 25\end{array}$

5.5.2 Aristotelian model, $r=3 \quad 25$

$\begin{array}{lll}\text { 5.5.3 Generic } r & 26\end{array}$

$\begin{array}{llr}6 & \text { Conclusion } & 27\end{array}$ 


\section{Introduction}

Tensor models [1] look superintegrable [12] and can be exactly solved [13, 14] by combinatorial methods [14-23] just like their matrix model prototypes [24]. However, original presentations [14-23] rely too much on the theory of symmetric groups and therefore can not attract much attention from physicists, generically unfamiliar with this subject. The goal of this note is to provide another formulation, which is much simpler. Following the strategy of $[12,13]$, we concentrate on the Gaussian averages, i.e. apply the standard functional integral technique of quantum field theory. As often happens, this eliminates unnecessary details and provides clear statements, while details can be restored afterwards. In this particular case, the main source of sophistication is the complicated structure of the space of gauge-invariant operators, but in fact many of them have vanishing Gaussian averages. Instead, the space of those with non-vanishing averages turns to be very simple, and it has a basis very similar to the basis of ordinary Schur functions. Thus, despite one does not expect straightforward applicability of the ordinary group theory in the tensor case, a very important sub-sector is actually controlled by something of this kind and can be investigated in depth. An option for the remaining part of the Hilbert space is a kind of non-linear realization as a qualitatively new extension of the idea of the separation into single- and multi-trace operators (while literally in the formalism of the present paper, the multi-trace operators for $r=2$ are treated on equal footing with the single-trace ones).

Our main idea in this paper is to concentrate on lifting to the tensor level of the main feature of matrix models, which, according to $[13,24]$, is that the average of linear group character in representation $R$ (the Schur function) is again a character:

$$
\left\langle\chi_{R}\right\rangle \sim \chi_{R}^{*}
$$

where the argument of character at the l.h.s. is the matrix-integration variable, while the character at the r.h.s. is taken on the "topological locus". Concrete expressions depend on the model (i.e. on the choice of the Vandermonde weight in the measure), and the suppressed $R$-dependent coefficient at the r.h.s., on its phase (i.e. the choice of the exponentiated Casimir weights in the measure, see [25-35] for the important notion of the phase in the theory of matrix-model and functional integrals). For examples of the different models in the Gaussian phase, see [36-38]. For examples beyond Gaussian phase, see [39].

In this paper, we start with the rectangular complex matrix model (RCM ) [40-42], which has a straightforward generalization to rainbow [13] tensor models [1]-[14], with well-studied Aristotelian (rank $r=3$ ) model [12] as the first non-trivial example. Our goal is to pose the problem of how (1.1) is generalized to $r>2$ and suggest a possible way to solve it. Deeper questions such as generalization of the group theory, which could underline the emerging structures are yet too early to address, and we leave them beyond the scope of the present paper. To avoid unnecessary complications, we consider only the Gaussian phase. 
In the case of RCM, eq. (1.1) can be written in full detail as

$$
\begin{aligned}
\frac{\int_{N_{1} \times N_{2}} \chi_{R}\left\{P_{k}=\operatorname{Tr}(M \bar{M})^{k}\right\} e^{-\operatorname{Tr} M \bar{M}} d^{2} M}{\int_{N_{1} \times N_{2}} e^{-\operatorname{Tr} M \bar{M}} d^{2} M} & =\left\langle\chi_{R}\left\{P_{k}\right\}\right\rangle=\frac{D_{R}\left(N_{1}\right) D_{R}\left(N_{2}\right)}{d_{R}} \\
& =\frac{\chi_{R}\left\{p_{k}=N_{1}\right\} \chi_{R}\left\{p_{k}=N_{2}\right\}}{d_{R}}
\end{aligned}
$$

where $\chi_{R}$ is the Schur function, which is a symmetric function of some variables $x_{i}$ (here the eigenvalues of the matrix $M \bar{M})$ or a function of time variables $p_{k}:=\sum_{i} x_{i}^{k}, d_{R}=$ $\chi_{R}\left(p_{k}=\delta_{1, k}\right)$. The integrals here are over $N_{1} \times N_{2}$ (rectangular) complex matrices $M$, the model has a "gauge" symmetry $\mathrm{U}\left(N_{1}\right) \times \mathrm{U}\left(N_{2}\right)$. The character (Schur function) depends on the Young diagram $R$ and on the sequence of time-variables $p_{k}$, e.g.

$$
\chi_{\emptyset}\{p\}=1, \quad \chi_{[1]}\{p\}=p_{1}, \quad \chi_{[2]}\{p\}=\frac{p_{2}+p_{1}^{2}}{2}, \quad \chi_{[1,1]}\{p\}=\frac{-p_{2}+p_{1}^{2}}{2}, \quad \ldots
$$

If one ascribes $p_{k}$ the grading $k$, then $\chi_{R}\{p\}$ is homogeneous of degree $|R|$, which is the size (number of boxes) of the diagram $R$. The role of time-variables at the l.h.s. in eq. (1.2) is played by the single-trace gauge invariants $P_{k} \equiv \operatorname{Tr}(M \bar{M})^{k}$, while at the r.h.s. they are fixed at the "classical topological locus", where all $p_{k}=N$. At these values, the character of the representation $R$ is equal to the dimension of the representation $R, D_{R}(N)$.

Now comes the first crucial observation: bilinear combination is not generic, from the entire set of all $\chi_{R_{1}}\left\{N_{1}\right\} \chi_{R_{2}}\left\{N_{2}\right\}$, only the subset $R_{2}=R_{1}$ is selected. If we look at the result of [12] for Aristotelian model, we easily observe that the same is true there: the set of averages of a given grading is not an arbitrary function of colorings $N_{1}, N_{2}, N_{3}$, but is restricted. Say, at level $n=2$, allowed at the r.h.s. are only

$$
\chi_{[2],[2],[2]}^{*} \quad \chi_{[2],[1,1],[1,1]}^{*} \quad \chi_{[1,1],[2],[1,1]}^{*} \quad \chi_{[1,1],[1,1],[2]}^{*}
$$

while the other four

$$
\chi_{[2],[2],[1,1]}^{*} \quad \chi_{[2],[1,1],[2]}^{*} \quad \chi_{[1,1],[1,1],[2]}^{*} \quad \chi_{[1,1],[1,1],[1,1]}^{*}
$$

are forbidden. We introduced here an abbreviated notation $\chi_{R_{1}, R_{2}, R_{3}}^{*}=$ $D_{R_{1}}\left(N_{1}\right) D_{R_{2}}\left(N_{2}\right) D_{R_{3}}\left(N_{3}\right)$. The second observation is that the allowed $\chi_{R_{1}, R_{2}, R_{3}}^{*}$ are actually averages of the operators $\chi_{R_{1}, R_{2}, R_{3}}$ which are of the symmetry type $R_{1} \otimes R_{2} \otimes R_{3}$ from the point of view of the group $S_{n}^{\otimes 3}$. Finally, the third observation is that an attempt to build up an operator $\chi_{R_{1}, R_{2}, R_{3}}$ of forbidden symmetry type gives zero, this is the reason why such $\chi_{R_{1}, R_{2}, R_{3}}$ does not emerge among averages.

Our goal in this text is to study and extend these observations to other gradings and ranks with an obvious purpose to understand the way to generalize (1.1) and, hence, the very notion of character (Schur functions) to tensor models. Though looking at the Gaussian averages is simpler than at the operators, we emphasize that the story is actually about operators $\mathcal{K}$, and, like in the matrix model case, it is essentially independent of the averaging procedure, in particular, of the choice of the Gaussian phase of the model. 
The main result of the present paper is that (1.2) has a direct generalization to the rainbow tensor models: there is a set of gauge invariant operators

$$
\chi_{R_{1}, \ldots, R_{r}}(M, \bar{M})=\frac{1}{n !} \sum_{\sigma_{1}, \ldots, \sigma_{r} \in S_{n}} \psi_{R_{1}}\left(\sigma_{1}\right) \ldots \psi_{R_{r}}\left(\sigma_{r}\right) \cdot \mathcal{K}_{\sigma_{1}, \ldots, \sigma_{r}}^{(n)}
$$

which are linear combinations of the tensorial counterparts of "multi-trace" operators

$$
\mathcal{K}_{\sigma_{1}, \ldots, \sigma_{r}}^{(n)}=\sum_{\vec{a}^{1}=1}^{N_{1}} \ldots \sum_{\vec{a}^{r}=1}^{N_{r}}\left(\prod_{p=1}^{n} M_{a_{p}^{1}, \ldots a_{p}^{r}} \bar{M}^{a_{\sigma_{1}(p)}^{1}, \ldots, a_{\sigma_{r}(p)}^{r}}\right)
$$

with the coefficients made from symmetric group characters $\psi_{R}(\sigma)$ such that their Gaussian averages with the weight $\exp \left(-\mathcal{K}_{i d, \ldots, i d}^{(1)}\right)=\exp \left(-\sum_{a^{1}=1}^{N_{1}} \ldots \sum_{a^{r}=1}^{N_{r}} M_{a^{1} \ldots a^{r}} \bar{M}^{a^{1} \ldots a^{r}}\right)$ are essentially the products of dimensions:

$$
\left\langle\chi_{R_{1}, \ldots, R_{r}}\right\rangle=C_{R_{1}, \ldots, R_{r}} \cdot \frac{D_{R_{1}}\left(N_{1}\right) \cdot \ldots \cdot D_{R_{r}}\left(N_{r}\right)}{d_{R_{1}} \cdot \ldots \cdot d_{R_{r}}}
$$

The sizes of all the Young diagrams $R_{k}$ are the same and equal to the power $n$ in $M$ and $\bar{M}$, which we call level in what follows. Our main claim is that $\chi_{\vec{R}}$ form the full basis of operators with non-vanishing Gaussian averages (see section 3.5). This basis is overcomplete, i.e. there are linear dependencies, but it is smaller than the full basis of all gauge invariant operators, which grows much faster than the number of $\chi_{\vec{R}}$ and even than the $r$-th power of the number of Young diagrams. The reason is that there are many operators with vanishing Gaussian averages. The coefficients $C_{\vec{R}}$ are $r$-counterparts of the Clebsch-Gordan coefficients, which, in fact, just select allowed symmetries. In the case of $r=2$, i.e. for the RCM, $C_{R_{1}, R_{2}}=d_{R_{1}} \delta_{R_{1}, R_{2}}$ and we return to (1.2). Actually, $\chi_{\vec{R}}$ itself is proportional to $C_{\vec{R}}$, i.e. it is the operator, not just the average, which vanishes in the case of forbidden symmetry.

Perhaps, a main problem with $\chi_{\vec{R}}$ is that, for $r>2$, they are too few, much less than the number of gauge-invariant operators, which are labeled by a peculiar double coset of symmetric group $S_{n} \backslash S_{n}^{r} / S_{n}$ [12]. Thus one can continue a search for more genuine tensorial characters, or at least for the coset ones. Unlike $\chi_{\vec{R}}$, which deserve the name Kronecker characters, generic tensorial and coset ones are not expected to be invariant under arbitrary conjugations: the symmetry of the coset is smaller. We do not go into details of this further generalization, the story of Kronecker characters $\chi_{\vec{R}}$ is already quite something to consume: an unexpected and far going generalization from matrices to tensors, see also [43].

Our main target in this paper is the Kronecker tensorial characters (1.3) and their properties. We start in section 2 with explaining the main idea in simple examples of first levels of the RCM and the Aristotelian $r=3$ model. Then, in section 3, we introduce formal definition of the generalized (Kronecker) characters and discuss their properties. Section 4 is devoted to the orthogonality of these characters, while, in section 5 , we introduce the generalized cut-and-join operators $\hat{W}$ that have the generalized characters as their eigenvalues. Section 6 contains some concluding remarks. 


\section{The main idea}

\subsection{Operators with a given symmetry}

Our main idea is to consider the basis of gauge invariant operators associated with elements of the tensor product of $r$ copies of the group algebras of symmetric group.

Consider an element of the group algebra of $S_{n}$,

$$
\hat{R}_{\alpha}=\sum_{\sigma \in S_{n}} \alpha_{\sigma} \cdot \hat{\sigma}
$$

where $\sigma$ are the elements of the symmetric group $S_{n}$. We now construct a basis in the space of operators $\mathcal{K}$ of level $n$, by the action of $r$ operations $\hat{R}_{1} \otimes \ldots \otimes \hat{R}_{r}$ on the operator $\mathcal{K}_{i d^{r}}^{n}$. For instance, for $r=3$

$$
\hat{R}_{\alpha} \otimes \hat{R}_{\alpha^{\prime}} \otimes \hat{R}_{\alpha^{\prime \prime}}=\sum_{\sigma} \alpha_{\sigma} \hat{\sigma} \otimes \sum_{\sigma} \alpha_{\sigma}^{\prime} \hat{\sigma} \otimes \sum_{\sigma} \alpha_{\sigma}^{\prime \prime} \hat{\sigma}
$$

acts on the operator $\mathcal{K}_{i d, i d, i d}^{n}=\prod_{p=1}^{n} M_{a_{p} b_{p} c_{p}} \bar{M}^{a_{p} b_{p} c_{p}}$ as follows:

$$
\hat{R}_{\alpha} \otimes \hat{R}_{\alpha^{\prime}} \otimes \hat{R}_{\alpha^{\prime \prime}}: \quad \mathcal{K}_{i d, i d, i d}^{n} \longrightarrow \chi_{\alpha, \alpha^{\prime}, \alpha^{\prime \prime}}=\sum_{\sigma, \sigma^{\prime}, \sigma^{\prime \prime} \in S_{n}} \alpha_{\sigma} \alpha_{\sigma^{\prime}}^{\prime} \alpha_{\sigma^{\prime \prime}}^{\prime \prime} \prod_{p=1}^{n} M_{a_{p} b_{p} c_{p}} \bar{M}^{a_{\sigma(p)} b_{\sigma^{\prime}(p)} c_{\sigma^{\prime \prime}(p)}}
$$

Now one can choose the coefficients $\alpha$ 's in such a way that they are associated with some symmetry patterns described by Young diagrams: with representations of $S_{n}$. For instance, up to the level $n=3$, there are 3 different patterns:

- $[n] \in S_{n}$ : symmetrization with all the equal weights of one unit

$$
\hat{S}=\sum_{\sigma \in S_{n}} \hat{\sigma}
$$

- $\left[1^{n}\right] \in S_{n}$ : antisymmetrization with weights depending on the parity of the permutation

$$
\hat{A}=\sum_{\sigma \in S_{n}}(-)^{P_{\sigma}} \hat{\sigma}
$$

- $[2,1] \in S_{3}$ : in this particular case we define

$$
\hat{B}=1-\hat{P}_{13}+\hat{P}_{12}-\hat{P}_{13} \hat{P}_{12}
$$

This definition follows the standard rule: make a Young tableau \begin{tabular}{|l|l|}
\hline 1 & 2 \\
\hline 3 & , first sym-
\end{tabular} metrize in rows and then antisymmetrize in columns:

$$
\hat{B} \psi^{a_{1} a_{2} a_{3}}=\psi^{a_{1} a_{2} a_{3}}+\psi^{a_{2} a_{1} a_{3}}-\psi^{a_{3} a_{2} a_{1}}-\psi^{a_{2} a_{3} a_{1}}
$$

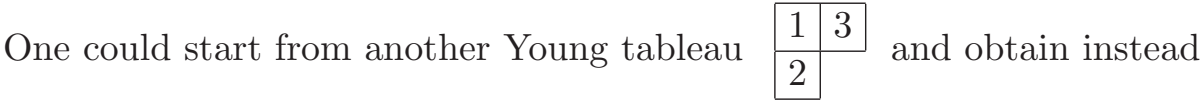

$$
\begin{aligned}
\hat{\tilde{B}} & =1-\hat{P}_{12}+\hat{P}_{13}-\hat{P}_{12} \hat{P}_{13} \\
\hat{\tilde{B}} \psi^{a_{1} a_{2} a_{3}} & =\psi^{a_{1} a_{2} a_{3}}+\psi^{a_{3} a_{2} a_{1}}-\psi^{a_{2} a_{1} a_{3}}-\psi^{a_{3} a_{1} a_{2}}
\end{aligned}
$$




\subsection{RCM}

Let us start with the $r=2$ case. If we consider averages (1.2) of a given grading, we obtain functions of $N_{1}$ and $N_{2}$, which are inhomogeneous, but not arbitrary:

- Level 1: $\operatorname{Im}_{1}^{(2)}=\operatorname{Span}\left\{D_{[1]}\left(N_{1}\right) D_{[1]}\left(N_{2}\right)=N_{1} N_{2}\right\}$

- Level 2: $\operatorname{Im}_{2}^{(2)}=\operatorname{Span}\left\{D_{[2]}\left(N_{1}\right) D_{[2]}\left(N_{2}\right)=N_{1} N_{2}\left(N_{1}+1\right)\left(N_{2}+1\right), D_{[1,1]}\left(N_{1}\right) D_{[1,1]}\left(N_{2}\right)\right.$ $\left.=N_{1} N_{2}\left(N_{1}-1\right)\left(N_{2}-1\right)\right\}$. However, neither $D_{[2]}\left(N_{1}\right) D_{[1,1]}\left(N_{2}\right)=N_{1} N_{2}\left(N_{1}+1\right)\left(N_{2}-1\right)$ nor $D_{[1,1]}\left(N_{1}\right) D_{[2]}\left(N_{2}\right)=N_{1} N_{2}\left(N_{1}-1\right)\left(N_{2}+1\right)$ belongs to $\operatorname{Im}_{2}^{(2)}$.

- Level $n$ : the same persists at higher levels: $\operatorname{Im}_{n}^{(2)}=\operatorname{Span}\left\{D_{R}\left(N_{1}\right) D_{R}\left(N_{2}\right), R \vdash n\right\}$, but none of non-diagonal $D_{R_{1}}\left(N_{1}\right) D_{R}\left(N_{2}\right)$ with $R_{1}, R_{2} \vdash n$ and $R_{2} \neq R_{1}$ belongs to this space.

We used here the standard notation $R \vdash n$, meaning that the size (number of boxes) in $R$ is $n$, i.e. $|R|=n$. Dimension of $\operatorname{Im}_{n}^{(2)}$ is just the number of Young diagrams of the size $n$, i.e.

$$
\sum_{n} \operatorname{dim}\left(\operatorname{Im}_{n}^{(2)}\right) \cdot q^{n}=\prod_{n} \frac{1}{1-q^{n}}
$$

The fact that the image is spanned by a given set labeled with $R_{1}=R_{2}=R$, means that one can enumerate and classify the averaged quantities: they are labeled by Young diagrams, and, indeed, they are just characters. Looking at the averages, one can determine that any product of characters is linearly expressed through characters themselves. This is a well known fundamental fact, but, what is important, one can now extract it from studying the averages, and this approach can be straightforwardly extendable from matrices to tensors, where we know neither what the characters are, nor their properties. Information that we need is just the structure of spaces $\operatorname{Im}_{n}^{(r)}$. Moreover, one can begin just from the linear space structure, namely from the basis in $\operatorname{Im}_{n}^{(r)}$ made from $r$-linear combinations of dimensions

$$
\chi_{\vec{R}}^{*}=\prod_{i=1}^{r} D_{R_{i}}\left(N_{i}\right)
$$

with all $R_{i} \vdash n$.

In these terms, the statement for RCM is that $\operatorname{Im}_{n}^{(2)}$ is spanned by the "diagonal" $\chi_{R, R}^{*}$, and these are averages of $\chi_{R}\{P\}$ with $P_{k}=\operatorname{Tr}(M \bar{M})^{k}$.

More than that, if we ask what could be the quantity, whose average would produce a non-diagonal $\chi_{R_{1}, R_{2}}^{*}$, the answer will be zero, this is why such averages do not actually arise. Indeed, operators in the RCM can be a priori labeled by two permutations $\sigma_{1}, \sigma_{2} \in S_{n}$ :

$$
\mathcal{O}_{\sigma_{1}, \sigma_{2}}=\prod_{p=1}^{n} M_{a_{p} b_{p}} \bar{M}^{a_{\sigma_{1}(p)} b_{\sigma_{2}(p)}}
$$


(summation is assumed over all $a_{p}$ and $b_{p}$ ). However, not all these operators are independent: in fact

$$
\begin{array}{rlrl}
n & =1 \\
n & =2 & \mathcal{O}_{(1),(1)} & =\operatorname{Tr} M \bar{M}=P_{1} \\
& \mathcal{O}_{(1)(2),(1)(2)} & =\mathcal{O}_{(12),(12)}=P_{1}^{2}, & \\
& & \mathcal{O}_{(1)(2),(12)}=\mathcal{O}_{(12),(1)(2)}=P_{2}
\end{array}
$$

so that for $S=(1)(2)+(12)=I+P$ and $A=(1)(2)-(12)=I-P$

$$
\mathcal{O}_{S S}=2\left(P_{2}+P_{1}^{2}\right)=4 \chi_{[2]}\{P\}, \quad \mathcal{O}_{A A}=2\left(-P_{2}+P_{1}^{2}\right)=4 \chi_{[11]}\{P\}
$$

while

$$
\mathcal{O}_{S A}=\mathcal{O}_{A S}=0
$$

In other words, the fact about the averages can be observed before averaging, i.e. is actually independent of the phase of the model. Thus we think that technically the simplest approach to study the space of gauge invariant operators is provided by the expression of Gaussian averages through dimensions $D_{R}(N)$.

\subsection{Aristotelian model}

In fact, all the information needed in this case can be extracted from the detailed study made in [12]. We just need to reformulate these results in terms which are relevant to purposes of the present paper. We refer to [12] for the tables of operators and the notation.

- Level 1: $\operatorname{dim}\left(\operatorname{Im}_{1}^{(3)}\right)=1$,

and the relevant character is $\chi_{[1],[1],[1]}^{*}=D_{[1]}\left(N_{1}\right) D_{[1]}\left(N_{2}\right) D_{\left[N_{3}\right]}=N_{1} N_{2} N_{3}$, the corresponding operator $\mathcal{K}_{1}=\sum_{a=1}^{N_{1}} \sum_{b=1}^{N_{2}} \sum_{c=1}^{N_{3}} M_{a b c} \bar{M}^{a b c}=M_{a b c} \bar{M}^{a b c}$ (hereafter, the summation over repeated indices $a, b, c, \ldots$ is assumed).

- Level 2: $\operatorname{dim}\left(\operatorname{Im}_{2}^{(3)}\right)=4$, and the relevant characters are

$$
\begin{aligned}
\chi_{S S S}^{*}=D_{[2]}\left(N_{1}\right) D_{[2]}\left(N_{2}\right) D_{[2]}\left(N_{3}\right) & =\frac{1}{8} N_{1} N_{2} N_{3}\left(N_{1}+1\right)\left(N_{2}+1\right)\left(N_{3}+1\right) \\
& =\frac{1}{8}\left\langle\mathcal{K}_{1}^{2}+\mathcal{K}_{2}+\mathcal{K}_{2}+\mathcal{K}_{2}\right\rangle \\
\chi_{S A A}^{*}=D_{[2]}\left(N_{1}\right) D_{[1,1]}\left(N_{2}\right) D_{[1,1]}\left(N_{3}\right) & =\frac{1}{8} N_{1} N_{2} N_{3}\left(N_{1}+1\right)\left(N_{2}-1\right)\left(N_{3}-1\right) \\
& =\frac{1}{8}\left\langle\mathcal{K}_{1}^{2}+\mathcal{K}_{2}-\mathcal{K}_{2}-\mathcal{K}_{2}\right\rangle \\
\chi_{A S A}^{*}=D_{[1,1]}\left(N_{1}\right) D_{[2]}\left(N_{2}\right) D_{[1,1]}\left(N_{3}\right) & =\frac{1}{8} N_{1} N_{2} N_{3}\left(N_{1}-1\right)\left(N_{2}+1\right)\left(N_{3}-1\right) \\
& =\frac{1}{8}\left\langle\mathcal{K}_{1}^{2}-\mathcal{K}_{2}+\mathcal{K}_{2}-\mathcal{K}_{2}\right\rangle \\
\chi_{A A S}^{*}=D_{[1,1]}\left(N_{1}\right) D_{[1,1}\left(N_{2}\right) D_{[2]}\left(N_{3}\right) & =\frac{1}{8} N_{1} N_{2} N_{3}\left(N_{1}-1\right)\left(N_{2}-1\right)\left(N_{3}+1\right) \\
& =\frac{1}{8}\left\langle\mathcal{K}_{1}^{2}-\mathcal{K}_{2}-\mathcal{K}_{2}+\mathcal{K}_{2}\right\rangle
\end{aligned}
$$


with $S=[2]$ and $A=[1,1]$. The operators $\mathcal{K}$ are defined in ([12], eq. (7.8) and appendix A.2). Thus, $\operatorname{Im}_{2}^{(3)}$ is spanned by $\chi_{S S S}^{*}$ and $3 \times \chi_{S A A}^{*}$, where 3 stands for the number of quantities obtained by permutations from $S_{3}$. Forbidden are the four other characters

$$
\chi_{S S A}^{*}=D_{[2]}\left(N_{1}\right) D_{[2]}\left(N_{2}\right) D_{[1,1]}\left(N_{3}\right)=\frac{1}{8} N_{1} N_{2} N_{3}\left(N_{1}+1\right)\left(N_{2}+1\right)\left(N_{3}-1\right),
$$

$\chi_{S A S}^{*}, \chi_{A S S}^{*}$ and $\chi_{A A A}^{*}$ : there are no such operators at level 2 of the Aristotelian model with appropriate discrete symmetry group properties.

- Level 3: $\operatorname{dim}\left(\operatorname{Im}_{3}^{(3)}\right)=11$, and the relevant operators are

$$
\chi_{S S S}, 3 \times \chi_{S A A}, 3 \times \chi_{S B B}, 3 \times \chi_{A B B}, \chi_{B B B}
$$

with $S=[3], B=[2,1], A=[1,1,1]$. Forbidden are $3 \times \chi_{S S A}, 3 \times \chi_{S S B}, 3 \times \chi_{A A B}$, $6 \times \chi_{S B A}$ and $\chi_{A A A}$.

Again, from analysis of averages, one obtains that, for instance,

$$
\chi_{S S S}^{*} \sim\left\langle\mathcal{K}_{1}^{3}+3\left(\mathcal{K}_{2}+\mathcal{K}_{2}+\mathcal{K}_{2}\right) \mathcal{K}_{1}+2\left(\mathcal{K}_{3}+\mathcal{K}_{3}+\mathcal{K}_{3}\right)+6\left(\mathcal{K}_{2,2}+\mathcal{K}_{2,2}+\mathcal{K}_{2,2}\right)+2 \mathcal{K}_{3 W}\right\rangle
$$

and the operator at the r.h.s. is exactly the triple symmetrization of $M_{a_{1} b_{1} c_{1}} M_{a_{2} b_{2} c_{2}} M_{a_{3} b_{3} c_{3}} \bar{M}^{a_{1} b_{1} c_{1}} \bar{M}^{a_{2} b_{2} c_{2}} \bar{M}^{a_{3} b_{3} c_{3}}$ w.r.t. indices $\left(a_{1}, a_{2}, a_{3}\right), \quad\left(b_{1}, b_{2}, b_{3}\right)$ and $\left(c_{1}, c_{2}, c_{3}\right)$ of $\bar{M}$ (they are defined in [12], eq. (7.19) and appendix A.3). Forbidden symmetrizations such as $S B A$ are vanishing already at the operator level.

The $r=3$ counterpart of $(2.8)$,

$$
\begin{aligned}
& \sum_{n} \operatorname{dim}\left(\operatorname{Im}_{n}^{(3)}\right) \cdot q^{n}=\prod_{n=1} \frac{1}{\left(1-q^{n}\right)^{\# \operatorname{dde}(\mathrm{n})}}= \\
& \quad=\prod_{n=1} \frac{1}{(1-q)\left(1-q^{2}\right)^{3}\left(1-q^{3}\right)^{7}\left(1-q^{4}\right)^{26}\left(1-q^{5}\right)^{97}\left(1-q^{6}\right)^{624}\left(1-q^{7}\right)^{4163} \cdots}
\end{aligned}
$$

appears to imply that the classification problem is hopeless. In fact, it was already addressed in [15-23] and [12], but in what follows we look at it from a somewhat different direction in the spirit of [44] and [43].

Note that the coefficients in (2.17) grow much faster than the triples of Young diagram (the cubes of the coefficients in (2.8)). This means that $\chi_{R_{1}, R_{2}, R_{3}}$ do not exhaust all gauge invariant operators. At the same time, as discovered in [12], the number of linearly independent Gaussian averages is lower than the prediction of (2.17), and it is actually less than the number of triples. This is why $\chi_{R_{1}, R_{2}, R_{3}}$ are enough to enumerate all operators with non-vanishing Gaussian average, though $\chi_{R_{1}, R_{2}, R_{3}}$ form an overfull basis. This is reflected in the fact that the triple products of dimensions are not all linearly independent. The differences between the number of all gauge invariant operators, of $\chi_{R_{1}, R_{2}, R_{3}}$ and of independent Gaussian averages all appear starting from level $n=4$. 
- Level $n=4$ : not all of the 43 linearly independent operators at this level have independent Gaussian averages: this was overlooked in [12], where the phenomenon was first observed only at level 5. In fact, independent are just 30 averages, while there are relations

$$
\begin{aligned}
<\mathcal{K}_{3 W} \mathcal{K}_{1}>+3<\mathcal{K}_{222}>+2<\mathcal{K}_{2}^{2}> & =2<\mathcal{K}_{3} \mathcal{K}_{1}>+2<\mathcal{K}_{2,2,2}>+2<\mathcal{K}_{2,2,2}> \\
<\mathcal{K}_{31 W}> & =<\mathcal{K}_{2,2} \mathcal{K}_{1}>-<\mathcal{K}_{2} \mathcal{K}_{2}>+<\mathcal{K}_{22 W}>
\end{aligned}
$$

plus four more relations obtained by cyclic permutations of colorings (these operators are defined in ([12], eq. (7.26) and appendix A.4). Thus, for instance, the combination

$$
\mathcal{K}_{31 W}-\mathcal{K}_{2,2} \mathcal{K}_{1}+\mathcal{K}_{2} \mathcal{K}_{2}-\mathcal{K}_{22 W}
$$

is a non-trivial operator, but its average vanishes. The 30-dimensional linear space of averages is spanned by 43 triple-characters $\chi_{R_{1}, R_{2}, R_{3}}^{*}$. They also produce an overfull basis in the space of operators with non-vanishing Gaussian averages.

We make a table for the Aristotelian model $(r=3)$ :

\begin{tabular}{c|ccc} 
Level & \# of gauge invariant operators \# of independent Gaussian averages \# of characters \\
\hline & & 1 & 1 \\
1 & 1 & 4 & 4 \\
2 & 4 & 11 & 11 \\
3 & 11 & 30 & 43 \\
4 & 43 & 61 & 143 \\
5 & 161 & & 511 \\
6 & 901 & & 1599
\end{tabular}

The first and the third columns coincide up to level 4 , because the operators $\chi_{R_{1}, R_{2}, R_{3}}$ are non-zero if and only if $C_{R_{1}, R_{2}, R_{3}}$ are non-zero (see section 3.4), $C_{R_{1}, R_{2}, R_{3}}$ being the Clebsch-Gordan coefficients for the symmetric group representations, while the number of gauge invariant operators is ([12], eq. (6.19))

$$
\#_{\text {g.-inv.ops }}=\sum_{R_{1}, R_{2}, R_{3} \vdash n} C_{R_{1} R_{2} R_{3}}^{2}
$$

and non-trivial multiplicities emerge starting from level 5. By this reason, the numbers in the first column are larger than in the third one at higher levels.

The numbers in the second column are smaller than those in the third one, which is evident for the following reason: the Gaussian averages are polynomials in $N_{i}$, with each $N_{i}$ entering with the maximal degree of $n$, and all averages are proportional to $N_{1} N_{2} N_{3}$. Hence, the linearly independent basis for the Gaussian averages is given, e.g., by monomials 
$N_{1}^{i_{1}} N_{2}^{i_{2}} N_{3}^{i_{3}}, i_{1}, i_{2}, i_{3}=1, \ldots n$, and, thus, the number of linearly independent polynomials at level $n$ is restricted by $n^{3}$ :

$$
\#_{\text {lin.indep.G.averages }} \leq n^{3}
$$

This number grows with $n$ much slower than the number of non-vanishing characters $\chi_{R_{1} R_{2} R_{3}}$, since the latter are labeled by triples of partitions of $n$. The number of partitions $P(n)$ at level $n$ grows much faster than $n$, and so does the number of triples $P(n)^{3}$. While one has to subtract from $P(n)^{3}$ the number of zero Clebsch-Gordan coefficients, this latter number grows slower ${ }^{1}$ than $P(n)^{3}$ at large $n$. Hence,

$$
\#_{C \neq 0}=\#_{\chi} \approx \#_{r-\text { tuples }} \quad \text { as } n \longrightarrow \infty
$$

Finally,

$$
\#_{\text {lin.indep.G.averages }} \leq \#_{\text {lin.indep. } \chi}
$$

and there is no equality beginning from $n=4$ : there are linear combinations of characters $\chi_{R_{1}, R_{2}, R_{3}}$ which are non-vanishing by themselves, but have vanishing Gaussian averages. See (2.19) for the first example.

Thus, we come to the following conclusion:

- The structures with allowed symmetries are the substitutes of characters, and their averages are products of the corresponding dimensions labeled by Young diagrams of size $n$.

- These characters are labeled by triples (generically, r-ples) of Young diagrams of size $n$ but not arbitrary: many triples provide vanishing operators.

\section{$3 \quad$ Generalized characters}

Now we give formal definitions of the generalized characters, that is, gauge invariant operators $\chi_{\vec{R}}\{M, \bar{M}\}$ as particular linear combinations of $\mathcal{K}$, and demonstrate that they and their Gaussian averages have nice properties, expected from the character-like quantities.

We also explain why these operators exhaust all which have non-vanishing averages.

\subsection{Some basic properties of symmetric group characters}

In what follows, we need characters $\psi_{R}(\gamma)$ of the permutation group $S_{n}$ (for the theory of permutation groups, see [45-47]). Let us note that the characters effectively depend only on the conjugation class $[\gamma]$ of the permutation: $\psi_{R}(\gamma)=\psi_{R}([\gamma])$. Hereafter, we denote by lower case Greek letters elements of the permutation group, and, by capital letters the

\footnotetext{
${ }^{1}$ For instance, the ratio $\xi_{n}:=\frac{\#_{C_{(n)}} \neq 0}{\#_{C_{(n)}}=0}$ behaves as a function of $n$ as

$$
\frac{\xi(n)}{n}=0.23,0.13,0.14,0.10,0.13,0.11,0.12,0.12,0.13,0.13 \quad \text { at } n=3, \ldots, 12 \text {. }
$$
}


conjugation classes (which are described by Young diagrams). The number of elements in the conjugacy class $\Delta$ is given by $n ! / z_{\Delta}$, where $z_{\Delta}$ is the order of automorphism of the corresponding Young diagram. We will also need the orthogonality condition

$$
\sum_{\gamma} \psi_{R}(\gamma) \psi_{Q}(\gamma \circ \sigma)=\sum_{\gamma} \psi_{R}\left(\gamma^{-1}\right) \psi_{Q}(\gamma \circ \sigma)=\frac{\psi_{R}(\sigma)}{d_{R}} \delta_{Q R}
$$

and the value of character on the unit element:

$$
\psi_{R}(i d)=\psi_{R}\left(\left[1^{|R|}\right]\right)=d_{R} \cdot|R| !
$$

In particular, it follows that

$$
\frac{1}{|R| !} \sum_{\gamma} \psi_{R}(\gamma) \psi_{Q}(\gamma)=\sum_{\Delta} \frac{\psi_{R}(\Delta) \psi_{Q}(\Delta)}{z_{\Delta}}=\frac{\psi_{R}(i d)}{d_{R}|R| !} \cdot \delta_{R Q}=\delta_{R Q}
$$

Eq. (3.1) implies a whole set of identities, and the simplest one is

$$
\sum_{\gamma} \psi_{R}\left(\sigma_{1} \circ \gamma \circ \sigma_{2} \circ \gamma^{-1}\right)=\frac{\psi_{R}\left(\sigma_{1}\right) \psi_{R}\left(\sigma_{2}\right)}{d_{R}}
$$

The simplest way to prove this identity is to note that the 1.h.s. depends only on the conjugacy class of $\sigma_{1}$ and, hence, one can make a "Fourier" transform from the conjugacy classes of $\sigma_{1}$ to the Young diagrams $Q$ given by the kernel $\psi_{Q}\left(\sigma_{1}\right)$ :

$$
\sum_{\sigma_{1}} \psi_{Q}\left(\sigma_{1}\right) \sum_{\gamma} \psi_{R}\left(\sigma_{1} \circ \gamma \circ \sigma_{2} \circ \gamma^{-1}\right) \stackrel{(3.1)}{=} \sum_{\gamma} \frac{\psi_{R}\left(\gamma \circ \sigma_{2} \circ \gamma^{-1}\right)}{d_{R}} \delta_{R Q}=\frac{|R| ! \psi_{R}\left(\sigma_{2}\right)}{d_{R}} \delta_{R Q}
$$

Similarly, the same Fourier transform of the r.h.s. of (3.4) gives

$$
\sum_{\sigma_{1}} \psi_{Q}\left(\sigma_{1}\right) \frac{\psi_{R}\left(\sigma_{1}\right) \psi_{R}\left(\sigma_{2}\right)}{d_{R}} \stackrel{(3.3)}{=} \frac{R ! \psi_{R}\left(\sigma_{2}\right)}{d_{R}} \delta_{R Q}
$$

The essential thing in this proof is that the Fourier transformation in this case has no kernel.

\subsection{RCM, $r=2$}

One of the possible definitions of the Schur functions, depending on the time-variables $p_{k}$, expresses them through the characters $\psi_{R}(\Delta)$ :

$$
\chi_{R}\{p\}=\sum_{\Delta \vdash|R|} \frac{\psi_{R}(\Delta)}{z_{\Delta}} p_{\Delta},
$$

where the sum goes over all Young diagrams $\Delta=\left\{\delta_{1} \geq \delta_{2} \geq \ldots \delta_{l_{\Delta}}>0\right\}=\left\{1^{m_{1}}, 2^{m_{2}}, \ldots\right\}$ of the same size $|\Delta| \equiv \delta_{1}+\delta_{2}+\ldots+\delta_{l}$ as $|R|$, the symmetry factor is $z_{\Delta}=\prod_{i} m_{i} ! \cdot i^{m_{i}}$, and $p_{\Delta}$ is a monomial $p_{\Delta} \equiv p_{\delta_{1}} p_{\delta_{2}} \ldots p_{\delta_{l}}$. The orthogonality of $\psi$

$$
\sum_{\Delta} \frac{\psi_{R}(\Delta) \psi_{R^{\prime}}(\Delta)}{z_{\Delta}}=\delta_{R, R^{\prime}}
$$


implies orthogonality of $\chi$

$$
\left\langle\hat{\chi}_{R} \mid \chi_{R^{\prime}}\right\rangle=\delta_{R, R^{\prime}}
$$

where $\left\langle\hat{p}_{\Delta} \mid p_{\Delta}^{\prime}\right\rangle=z_{\Delta} \delta_{\Delta, \Delta^{\prime}}$, i.e. $\hat{p}_{\Delta}=z_{\Delta} \frac{\partial}{\partial p_{\delta_{1}}} \ldots \frac{\partial}{\partial p_{\delta_{l}}}$.

Gauge invariant operators in RCM are

$$
\mathcal{K}_{\sigma_{1}, \sigma_{2}}=\prod_{p=1}^{n} M_{a_{p} b_{p}} \bar{M}^{a_{\sigma_{1}(p)} b_{\sigma_{2}(p)}}=\mathcal{K}_{i d, \sigma_{1}^{-1} \circ \sigma_{2}}
$$

Here $\sigma_{i}$ are elements of the permutation group $S_{n}$. In fact, $\mathcal{K}_{i d, \sigma}$ depends only on the conjugacy class of $\sigma$,

$$
\mathcal{K}_{i d, \sigma}=P_{\Delta}
$$

with $P_{k}=\operatorname{Tr}(M \bar{M})^{k}$. We can now introduce a "Fourier transform"

$$
\begin{aligned}
& \chi_{R_{1}, R_{2}} \equiv \frac{1}{n !} \sum_{\sigma_{1}, \sigma_{2} \in S_{n}} \psi_{R_{1}}\left(\sigma_{1}\right) \psi_{R_{2}}\left(\sigma_{2}\right) \mathcal{K}_{\sigma_{1}, \sigma_{2}}=\frac{1}{n !} \sum_{\sigma_{1}, \sigma_{2} \in S_{n}} \psi_{R_{1}}\left(\sigma_{1}\right) \psi_{R_{2}}\left(\sigma_{2}\right) \mathcal{K}_{i d, \sigma_{1}^{-1} \circ \sigma_{2}} \stackrel{\sigma_{2} \rightarrow \sigma_{1} \circ \sigma_{2}}{=} \\
& =\frac{1}{n !} \sum_{\sigma_{1}, \sigma_{2} \in S_{n}} \psi_{R_{1}}\left(\sigma_{1}\right) \psi_{R_{2}}\left(\sigma_{1} \circ \sigma_{2}\right) \mathcal{K}_{i d, \sigma_{2}} \stackrel{(3.1)}{=} \frac{1}{n !} \cdot \frac{\delta_{R_{1}, R_{2}}}{d_{R_{1}}} \sum_{\sigma_{2}} \psi_{R_{2}}\left(\sigma_{2}\right) \mathcal{K}_{i d, \sigma_{2}}=\frac{\delta_{R_{1}, R_{2}}}{d_{R_{1}}} \chi_{R_{2}}\{P\}
\end{aligned}
$$

where at the last stage we used the fact that $\mathcal{K}_{\sigma}$ depends only on the conjugacy class $\Delta$ of $\sigma$ so that

$$
\frac{1}{n !} \sum_{\sigma} \psi_{R}(\sigma) \mathcal{K}_{i d, \sigma}=\sum_{\Delta \vdash n} \frac{\psi_{R}(\Delta)}{z_{\Delta}} \mathcal{K}_{i d, \sigma} \stackrel{(3.11)}{=} \sum_{\Delta \vdash n} \frac{\psi_{R}(\Delta)}{z_{\Delta}} P_{\Delta} \stackrel{(3.7)}{=} \chi_{R}\{P\}
$$

The transformation (3.12) is not invertible, if considered as a map from the space of functions $F_{\sigma_{1}, \sigma_{2}}$ to $\chi_{R_{1}, R_{2}}$, because the space of all possible $R$ at fixed $|R|=n$ and that of all possible $\sigma$ in $S_{n}$ have different dimensions: the number of $\sigma$, which is equal to $n$ !, is larger than the number of Young diagrams that enumerate the conjugacy classes of $\sigma$. However, the actual operators $\mathcal{K}_{\sigma_{1}, \sigma_{2}}$ actually depend only on the product $\sigma^{-1} \circ \sigma_{2}$, i.e. only on its conjugacy class. Thus, they are in one-to-one correspondence with characters, and are diagonal, $\chi_{R_{1}, R_{2}} \sim \delta_{R_{1}, R_{2}}$.

To summarize, our $\chi_{R_{1}, R_{2}}$ is essentially the character:

$$
\chi_{R_{1}, R_{2}}=\frac{\delta_{R_{1}, R_{2}}}{d_{R_{1}}} \chi_{R_{1}}\{P\}
$$

and its Gaussian average is fully symmetric in $R_{1}$ and $R_{2}$ :

$$
\left\langle\chi_{R_{1}, R_{2}}\right\rangle=\delta_{R_{1}, R_{2}} \frac{D_{R_{1}}\left(N_{1}\right) D_{R_{2}}\left(N_{2}\right)}{d_{R_{1}} d_{R_{2}}}
$$

An advantage of this redefinition is that now it can be straightforwardly generalized beyond $r=2$, i.e. from matrix to tensor models. 


\subsection{Aristotelian model, $r=3$}

Consider the Aristotelian $r=3$ case. Denote

$$
\mathcal{K}_{\sigma_{1} \sigma_{2} \sigma_{3}}=\prod_{p=1}^{n} M_{a_{p} b_{p} c_{p}} \bar{M}^{a_{\sigma_{1}(p)} b_{\sigma_{2}(p)} c_{\sigma_{3}(p)}}
$$

(as usual, summation is assumed over all $a_{p}, b_{p}$ and $c_{p}$ ). Now, one can introduce the quantity

$$
\chi_{R_{1} R_{2} R_{3}}:=\frac{1}{n !} \sum_{\left\{\sigma_{i}\right\} \in S_{n}} \psi_{R_{1}}\left(\sigma_{1}\right) \psi_{R_{2}}\left(\sigma_{2}\right) \psi_{R_{3}}\left(\sigma_{3}\right) \cdot \mathcal{K}_{\sigma_{1} \sigma_{2} \sigma_{3}}
$$

which generalizes the notion of character.

Unlike in RCM with $r=2$, already in the Aristotelian case of $r=3$ the sum can not be reduced to summation over the conjugation classes only. What remains is the sum over the elements of (the orbit of) the permutation group: in this case, the counterpart of (3.12) is

$$
\begin{aligned}
\chi_{R_{1} R_{2} R_{3}} & =\frac{1}{n !} \sum_{\left\{\sigma_{1}, \sigma_{2}, \sigma_{3}\right\} \in S_{n}} \psi_{R_{1}}\left(\sigma_{1}\right) \psi_{R_{2}}\left(\sigma_{2}\right) \psi_{R_{3}}\left(\sigma_{3}\right) \mathcal{K}_{\sigma_{1} \sigma_{2} \sigma_{3}} \\
& =\frac{1}{n !} \sum_{\left\{\sigma_{1}, \sigma_{2}, \sigma_{3}\right\} \in S_{n}} \psi_{R_{1}}\left(\sigma_{1}\right) \psi_{R_{2}}\left(\sigma_{1} \circ \sigma_{2}\right) \psi_{R_{3}}\left(\sigma_{1} \circ \sigma_{3}\right) \mathcal{K}_{i d, \sigma_{2}, \sigma_{3}}
\end{aligned}
$$

where we used the property $\mathcal{K}_{\sigma_{1} \sigma_{2} \sigma_{3}}=\mathcal{K}_{i d, \sigma_{1}^{-1} \circ \sigma_{2}, \sigma_{1}^{-1} \circ \sigma_{3}}$ which follows from the definition (3.16). In fact, there is a larger symmetry: one can preserve the form $\mathcal{K}_{i d, \sigma_{2}, \sigma_{3}}$, while making the conjugation $\sigma_{2} \rightarrow \gamma^{-1} \circ \sigma_{2} \circ \gamma, \sigma_{3} \rightarrow \gamma^{-1} \circ \sigma_{3} \circ \gamma$. We will ignore this fact in what follows. In any case, the transformation (3.17) is not one-to-one on any reasonable space, both on the left and on the right. This means that our $\chi_{R_{1} R_{2} R_{3}}$ is both insufficient to describe the entire space of gauge invariant operators and redundant to describe the space of of gauge invariant operators with non-vanishing Gaussian averages. It instead serves as a generalized character $\chi_{\vec{R}}$, which is simple by itself and closed under simple operations.

In fact, as we saw in examples in the previous sections, there is a much stronger statements: that $\left\langle\chi_{R_{1}, R_{2}, R_{3}}\right\rangle$ form a (redundant) basis in the space of all Gaussian averages. Taking an explicit expression for the Gaussian average of $\mathcal{K}_{\sigma_{1} \sigma_{2} \sigma_{3}}$ from [13, 14], we obtain:

$$
\left\langle\mathcal{K}_{\sigma_{1} \sigma_{2} \sigma_{3}}\right\rangle=\sum_{\substack{\left\{Q_{i}\right\} \vdash n \\ \gamma \in S_{n}}} \prod_{i=1}^{3} D_{Q_{i}}\left(N_{i}\right) \psi_{Q_{i}}\left(\gamma \circ \sigma_{i}\right)
$$

and, using the orthogonality condition (3.1), one immediately obtains (see also [44], eq. (79))

$$
\left\langle\chi_{R_{1} R_{2} R_{3}}\right\rangle=C_{R_{1} R_{2} R_{3}} \cdot \frac{D_{R_{1}}\left(N_{1}\right)}{d_{R_{1}}} \frac{D_{R_{2}}\left(N_{2}\right)}{d_{R_{2}}} \frac{D_{R_{3}}\left(N_{3}\right)}{d_{R_{3}}}
$$

where

$$
C_{R_{1} R_{2} R_{3}}:=\frac{1}{n !} \sum_{\gamma \in S_{n}} \psi_{R_{1}}(\gamma) \psi_{R_{2}}(\gamma) \psi_{R_{3}}(\gamma)=\sum_{\Delta \vdash n} \frac{\psi_{R_{1}}(\Delta) \psi_{R_{2}}(\Delta) \psi_{R_{3}}(\Delta)}{z_{\Delta}}
$$

are the Clebsch-Gordan coefficients, which vanish in the case of forbidden symmetries. 


\subsection{Vanishing of $\chi_{\vec{R}}$ with forbidden symmetries}

Let us prove that vanishing the Clebsch-Gordan coefficients implies not only vanishing of the Gaussian averages, but also the generalized characters $\chi_{\vec{R}}$ themselves.

The coefficients

$$
C_{R_{1}, R_{2}, R_{3}}^{\sigma_{2}, \sigma_{3}}:=\frac{1}{n !} \sum_{\gamma \in S_{n}} \psi_{R_{1}}(\gamma) \psi_{R_{2}}\left(\gamma \circ \sigma_{2}\right) \psi_{R_{3}}\left(\gamma \circ \sigma_{3}\right)
$$

in the definition (3.18) of $\chi_{R_{1} R_{2} R_{3}}$ satisfy the orthogonality condition

$$
\sum_{\sigma_{1}, \sigma_{2}} C_{R_{1} R_{2} R_{3}}^{\sigma_{1} \sigma_{2}} C_{Q_{1} Q_{2} Q_{3}}^{\sigma_{1} \sigma_{2}}=\frac{C_{R_{1} R_{2} R_{3}}}{d_{R_{1}} d_{R_{2}} d_{R_{3}}} \delta_{R_{1} Q_{1}} \delta_{R_{2} Q_{2}} \delta_{R_{3} Q_{3}}
$$

This means that

$$
\sum_{\sigma_{1}, \sigma_{2}}\left(C_{R_{1} R_{2} R_{3}}^{\sigma_{1} \sigma_{2}}\right)^{2}=\frac{C_{R_{1} R_{2} R_{3}}}{d_{R_{1}} d_{R_{2}} d_{R_{3}}}
$$

and, since at the l.h.s. we have a sum of squares of rational real-valued quantities, we get as an immediate corollary

$$
C_{R_{1} R_{2} R_{3}}=0 \Longrightarrow \text { all } C_{R_{1} R_{2} R_{3}}^{\sigma_{1} \sigma_{2}}=0 \stackrel{(3.18)}{\Longrightarrow} \chi_{R_{1} R_{2} R_{3}}=0
$$

Thus we proved that vanishing of the Clebsh-Gordan coefficient $C_{R_{1} R_{2} R_{3}}=0$ implies identical vanishing of the corresponding generalized character $\chi_{R_{1} R_{2} R_{3}}$.

\subsection{Generalized characters as a basis for operators with non-vanishing Gaus- sian averages}

Using the identity (3.25), we can now prove that the generalized characters form a basis in the space of all operators with non-vanishing Gaussian averages. To this end, it is enough to prove that any Gaussian average can be written as a linear combination of the generalized characters. Indeed, any Gaussian average is given by formula (3.19). In fact, as we already discussed, it is enough to consider the average of $\mathcal{K}_{i d, \sigma_{2} \sigma_{3}}$,

$$
\left\langle\mathcal{K}_{i d, \sigma_{2} \sigma_{3}}\right\rangle=\sum_{\substack{\left\{R_{i}\right\} \vdash n \\ \gamma \in S_{n}}} C_{R_{1} R_{2} R_{3} \sigma_{3}}^{\sigma_{3}} \prod_{i=1}^{3} D_{R_{i}}\left(N_{i}\right)
$$

In the sum, contribute only $R_{i}$ such that $C_{R_{1} R_{2} R_{3}}^{\sigma_{1} \sigma_{2}} \neq 0$, which, as follows from (3.25), simultaneously implies $C_{R_{1} R_{2} R_{3}} \neq 0$. In this case, one obtains from (3.20) that

$$
\left\langle\mathcal{K}_{i d, \sigma_{2} \sigma_{3}}\right\rangle=\sum_{\substack{\left\{R_{i}\right\} \vdash n \\ \gamma \in S_{n}}} C_{R_{1} R_{2} R_{3}}^{\sigma_{2} \sigma_{3}} \frac{d_{R_{1}} d_{R_{2}} d_{R_{3}}}{C_{R_{1} R_{2} R_{3}}}\left\langle\chi_{R_{1} R_{2} R_{3}}\right\rangle
$$

i.e. any Gaussian average can be, indeed, written as a linear combination of the generalized characters. Moreover, since the coefficients in this combination does not depend on $N_{i}$ 's, 
and the latter enter only through the generalized characters, this implies that the generalized characters form a basis in the space of all gauge invariant operators with non-vanishing Gaussian averages.

Let us point out that we understand by the space of all gauge invariant operators with non-vanishing Gaussian averages the space with two operators equivalent if their difference is an operator with vanishing Gaussian average. In fact, the Gaussian averages of the operators $\mathcal{K},(3.16)$ associated with concrete permutations are non-vanishing, which follows both from the explicit examples of ([12], eqs. (7.9), (7.15), s.7.4.1, etc.) and from associating the large $N$ limit of these Gaussian averages with (non-vanishing) Feynman diagrams in the matrix model [48]. However, the Gaussian averages of these operators are subject to vanishing linear combinations, and all of them are spanned by the generalized characters up to operators with vanishing Gaussian averages.

\subsection{Background metrics}

For the action $M_{a b c} \bar{M}^{\bar{a} \bar{b} \bar{c}} A_{\bar{a}}^{a} B_{\bar{b}}^{b} C_{\bar{c}}^{c}$, we get a generalization of the "cut" relation (3.20)

$$
\left\langle\chi_{R_{1} R_{2} R_{3}}\right\rangle=C_{R_{1} R_{2} R_{3}} \frac{\chi_{R_{1}}[A] \chi_{R_{2}}[B] \chi_{R_{3}}[C]}{d_{R_{1}} d_{R_{2}} d_{R_{3}}}
$$

Another matrix model relation ("join"),

$$
\left\langle\chi_{R}[M C] \chi_{R^{\prime}}[\bar{M} D]\right\rangle=\frac{\chi_{R}[C D]}{d_{R}} \cdot \delta_{R, R^{\prime}}
$$

seems not to have a direct generalization to $r>2$.

\subsection{Other rainbow models, arbitrary $r$}

Similarly, for arbitrary $r$, we define

$$
\chi_{R_{1}, \ldots, R_{r}}=\frac{1}{n !} \sum_{\sigma_{1}, \ldots, \sigma_{r} \in S_{n}} \psi_{R_{1}}\left(\sigma_{1}\right) \ldots \psi_{R_{r}}\left(\sigma_{r}\right) \mathcal{K}_{\sigma_{1}, \ldots, \sigma_{r}}
$$

where, as a generalization of (3.16),

$$
\mathcal{K}_{\vec{\sigma}}=\mathcal{K}_{\sigma_{1} \ldots \sigma_{r}}=\prod_{p=1}^{n} M_{a_{p}^{(1)} \ldots a_{p}^{(r)}} \bar{M}^{a_{\sigma_{1}(p)}^{(1)} \ldots a_{\sigma_{r}(p)}^{(r)}}=\prod_{p=1} M_{\vec{a}_{p}} \bar{M}^{\vec{a}_{\vec{\sigma}(p)}}
$$

The Gaussian averages are

$$
\sum_{\vec{\mu}}\left(\left\langle\mathcal{K}_{\vec{\mu}}\right\rangle \cdot \prod_{i=1}^{r} \psi_{R_{i}}\left(\mu_{i}\right)\right)=C_{\vec{R}} \cdot \prod_{i=1}^{r} \frac{D_{R_{i}}\left(N_{i}\right)}{d_{R_{i}}}
$$

where

$$
C_{\vec{R}}:=\frac{1}{n !} \sum_{\gamma \in S_{n}} \prod_{i=1}^{r} \psi_{R_{i}}(\gamma)=\sum_{\Delta \vdash n} \frac{\prod_{i=1}^{r} \psi_{R_{i}}(\Delta)}{z_{\Delta}}
$$

These coefficients can be also expressed through the Clebsch-Gordan coefficients (3.21):

$$
C_{\vec{R}}=\sum_{\left\{Q_{i}\right\}} C_{R_{1} R_{2} Q_{1}} C_{Q_{1} R_{3} Q_{2}} C_{Q_{2} R_{4} Q_{3}} \ldots C_{Q_{r-3} R_{r-1} R_{r}}
$$




\section{Orthogonality of generalized characters}

\subsection{The problem}

The first essential property of characters is orthogonality. Conventional characters satisfy the orthogonality condition [49]:

$$
\left\langle\chi_{R} \mid \chi_{R^{\prime}}\right\rangle=\delta_{R R^{\prime}}
$$

where the scalar product is explicitly given by

$$
\left\langle\chi_{R} \mid \chi_{R^{\prime}}\right\rangle:=\left.\chi_{R}\left(k \partial_{k}\right) \chi_{R^{\prime}}\left(p_{k}\right)\right|_{p_{k}=0}
$$

Indeed, parameterizing the Young diagram $\Delta$ by the numbers $m_{i}$ which counts the number of lines of the same length $i$ in the diagram (i.e., for instance, for $\Delta=[4,2,2,1], m_{1}=1$, $m_{2}=2, m_{3}=0$, and $m_{4}=1$ ) and taking into account that, in these terms, $z_{\Delta}=$ $\prod_{i: m_{i} \neq 0} i^{m_{i}} m_{i}$ ! with the product over all $i$ with non-zero $m_{i}$, one obtains

$$
\begin{aligned}
\left.\chi_{R}\left\{k \frac{\partial}{\partial p_{k}}\right\} \chi_{R^{\prime}}\left\{p_{k}\right\}\right|_{p_{k}=0} & =\sum_{\Delta, \Delta^{\prime}} \frac{\psi_{R}(\Delta)}{z_{\Delta}} \frac{\psi_{R^{\prime}}\left(\Delta^{\prime}\right)}{z_{\Delta^{\prime}}} \prod_{j}\left(j \frac{\partial}{\partial p_{j}}\right)^{m_{j}} \prod_{i} p_{i}^{m_{j}^{\prime}} \longrightarrow \\
\stackrel{p_{k}=0}{\longrightarrow} \sum_{\Delta, \Delta^{\prime}} \frac{\psi_{R}(\Delta)}{z_{\Delta}} \frac{\psi_{R^{\prime}}\left(\Delta^{\prime}\right)}{z_{\Delta^{\prime}}} \prod_{i: m_{i} \neq 0} i^{m_{i}} m_{i} ! \delta_{m_{i}, m_{i}^{\prime}} & =\sum_{\Delta, \Delta^{\prime}} \frac{\psi_{R}(\Delta)}{z_{\Delta}} \frac{\psi_{R^{\prime}}\left(\Delta^{\prime}\right)}{z_{\Delta^{\prime}}} z_{\Delta} \delta_{\Delta \Delta^{\prime}} \\
& =\sum_{\Delta} \frac{\psi_{R}(\Delta) \psi_{R^{\prime}}(\Delta)}{z_{\Delta}}=\delta_{R R^{\prime}}
\end{aligned}
$$

where, in the last equality, we have used the orthogonality relation (3.3). Thus, orthogonality of $\chi$ is essentially reduced to orthogonality of $\psi$.

However, above manipulation heavily depends on existence of $p$-variables, moreover, involve derivatives with respect to $p$. In this form, it has low chances for tensorial generalization. Fortunately, in [36] the first step was done towards elimination of $p$-variables and reformulation of the theory of $W$-operators directly in terms of $\chi$-variables. In what follows, we introduce an even more powerful and straightforward formalism, which remains to be properly understood, but is already sufficient for tensor model applications.

It involves two ideas. First, we substitute the monomials $p_{\Delta}$ by independent linear variables $\xi_{\Delta}$. Second, we extend this set of variables to $\xi_{\sigma}$ depending on permutations $\sigma$ rather than on their conjugation classes. Then, the dual characters, which were obtained by the substitution $p_{k} \longrightarrow k \partial / \partial p_{k}$, where the coefficient $k$ somehow "remembers" about non-linearity in $p$, are described by a linear substitution $\xi_{\sigma} \longrightarrow \partial / \partial \xi_{\sigma}$ without any $\sigma$ dependent coefficients. This is already nice, but most important, this formalism continues to work for tensors. Moreover, the apparent redundancy of the $\xi_{\sigma}$-variables turns out to be exactly what is needed to capture the set of $\mathcal{K}_{\vec{\sigma}}$ with non-vanishing Gaussian averages.

\subsection{RCM, $r=2$, through ordinary characters}

Following this plan, we substitute

$$
\chi_{R}\{p\}=\sum_{\Delta \vdash|R|} \frac{\psi_{R}(\Delta)}{z_{\Delta}} p_{\Delta} \longrightarrow \tilde{\chi}_{R}(\xi)=\sum_{\sigma \in S_{n}} \psi_{R}(\sigma) \cdot \xi_{\sigma}
$$


where $n=|R|$ and $\psi_{R}(\sigma):=\psi_{R}([\sigma])$ depends only on the conjugation class $\Delta=[\sigma]$ of the permutation $\sigma$. Since there are $\frac{n !}{z_{\Delta}}$ different permutations in this class, we read from these relations and (3.12) that

$$
\sum_{\sigma \in \Delta} \xi_{\sigma}=\frac{1}{n !} \sum_{\sigma \in \Delta} \mathcal{K}_{i d, \sigma}=\frac{1}{z_{\Delta}} P_{\Delta}
$$

Then, since

$$
\begin{aligned}
\left\langle p_{\Delta} \mid p_{\Delta^{\prime}}\right\rangle & =\left\langle p_{1}^{m_{1}} p_{2}^{m_{2}} p_{3}^{m_{3}} \ldots \mid p_{1}^{m_{1}^{\prime}} p_{2}^{m_{2}^{\prime}} p_{3}^{m_{3}^{\prime}} \ldots\right\rangle \\
& =\left.\partial_{p_{1}}^{m_{1}}\left(2 \partial_{p_{2}}\right)^{m_{2}}\left(3 \partial_{p_{3}}\right)^{m_{3}} \ldots p_{1}^{m_{1}^{\prime}} p_{2}^{m_{2}^{\prime}} p_{3}^{m_{3}^{\prime}} \cdots\right|_{p=0}=z_{\Delta} \delta_{\Delta, \Delta^{\prime}}
\end{aligned}
$$

it is natural to postulate a new scalar product for linear functions of $\xi$-variables:

$$
\left\langle\xi_{\sigma} \mid \xi_{\sigma^{\prime}}\right\rangle=\frac{\delta_{\sigma \sigma^{\prime}}}{n !}
$$

Indeed, in this case

$$
\left\langle P_{\Delta} \mid P_{\Delta^{\prime}}\right\rangle=z_{\Delta} z_{\Delta^{\prime}} \sum_{\sigma \in \Delta} \sum_{\sigma^{\prime} \in \Delta^{\prime}}\left\langle\left\langle\xi_{\sigma} \mid \xi_{\sigma^{\prime}}\right\rangle=z_{\Delta} z_{\Delta^{\prime}} \delta_{\Delta, \Delta^{\prime}} \sum_{\sigma \in \Delta} \frac{1}{|\sigma| !}=z_{\Delta} \delta_{\Delta, \Delta^{\prime}}\right.
$$

Then we immediately obtain

$$
\left\langle\tilde{\chi}_{R} \mid \tilde{\chi}_{R^{\prime}}\right\rangle=\sum_{\sigma, \sigma^{\prime} \in S_{n}} \psi_{R}(\sigma) \psi_{R^{\prime}}\left(\sigma^{\prime}\right)\left\langle\left\langle\xi_{\sigma} \mid \xi_{\sigma^{\prime}}\right\rangle=\frac{1}{n !} \sum_{\sigma \in S_{n}} \psi_{R}(\sigma) \psi_{R^{\prime}}(\sigma) \stackrel{(3.3)}{=} \delta_{R, R^{\prime}}\right.
$$

\subsection{RCM, $r=2$, through bi-characters}

If we keep in mind our goal of generalization from matrix to tensor models, we need now to rewrite the previous section in terms of the generalizable object, the bi-character $\chi_{R_{1}, R_{2}}$ instead of the ordinary one $\chi_{R}$. The natural counterpart of (4.4) would be

$$
\tilde{\chi}_{R_{1}, R_{2}}(\eta):=\sum_{\sigma_{1}, \sigma_{2} \in S_{n}} \psi_{R_{1}}\left(\sigma_{1}\right) \psi_{R_{2}}\left(\sigma_{2}\right) \cdot \eta_{\sigma_{1}, \sigma_{2}}
$$

with new indeterminants $\eta$. However, this degree of redundancy is too much even for tensor model generalizations. We can safely demand that $\eta$ like $\mathcal{K}$ operators is invariant under simultaneous conjugations, $\eta_{\sigma_{1}, \sigma_{2}}=\eta_{\sigma \circ \sigma_{1} \circ \sigma^{-1}, \sigma \circ \sigma_{2} \circ \sigma^{-1}}$, what allows one to substitute $\eta_{\sigma_{1}, \sigma_{2}}=\eta_{i d, \sigma_{1}^{-1} \circ \sigma_{2}}$, which is essentially the $\xi$-variables.

Namely, using (3.12) and (4.4), introduce (hereafter, we use the notation $\tilde{\chi}$ for the generalized character in $\xi$-variables)

$$
\tilde{\chi}_{R_{1} R_{2}}(\xi)=\frac{\delta_{R_{1} R_{2}}}{d_{R_{1}}} \sum_{\sigma \in S_{n}} \psi_{R_{2}}(\sigma) \cdot \xi_{\sigma}
$$

where $\left|R_{1}\right|=\left|R_{2}\right|=n$ and the scalar product is (4.7). Then

$$
\left\langle\tilde{\chi}_{R_{1} R_{2}} \mid \tilde{\chi}_{R_{1}^{\prime} R_{2}^{\prime}}\right\rangle=\frac{\delta_{R_{1} R_{2}} \delta_{R_{1}^{\prime} R_{2}^{\prime}}}{d_{R_{1}} d_{R_{1}^{\prime}}} \cdot \frac{1}{n !} \sum_{\gamma \in S_{n}} \psi_{R_{2}}(\gamma) \psi_{R_{2}^{\prime}}(\gamma) \stackrel{(3.3)}{=} \frac{\delta_{R_{1} R_{2}} \delta_{R_{1}^{\prime} R_{2}^{\prime}} \delta_{R_{1} R_{1}^{\prime}}}{d_{R_{1}}^{2}}
$$


One can also realize the scalar product (4.7) similarly to (4.2):

$$
\left\langle\tilde{\chi}_{R R} \mid \tilde{\chi}_{R^{\prime} R^{\prime}}\right\rangle:=\left.\frac{1}{|R| ! \cdot d_{R} d_{R^{\prime}}} \cdot \tilde{\chi}_{R}\left(\frac{\partial}{\partial \xi}\right) \tilde{\chi}_{R^{\prime}}(\xi)\right|_{\xi=0}
$$

where $\tilde{\chi}_{R}(\xi)$ is defined in (4.4).

For example, at level $n=2$

$$
\begin{gathered}
\tilde{\chi}_{[2],[2]} \stackrel{(3.12)}{=} \mathcal{K}_{i d, i d}+\mathcal{K}_{i d,(12)}=P_{1}^{2}+P_{2}=2 \tilde{\chi}_{[2]} \stackrel{(4.11)}{=} 2\left(\xi_{i d}+\xi_{(12)}\right) \\
\tilde{\chi}_{[1,1],[1,1]} \stackrel{(3.12)}{=} \mathcal{K}_{i d, i d}-\mathcal{K}_{i d,(12)}=P_{1}^{2}-P_{2}=2 \tilde{\chi}_{[1,1]} \stackrel{(4.11)}{=} 2\left(\xi_{i d}-\xi_{(12)}\right)
\end{gathered}
$$

and

$$
\begin{aligned}
\left\langle\tilde{\chi}_{[1,1],[1,1]} \mid \tilde{\chi}_{[1,1],[1,1]}\right\rangle & =4\left\langle\left\langle\tilde{\chi}_{[1,1]} \mid \tilde{\chi}_{[1,1]}\right\rangle=\frac{2^{2}}{2 !}\left(\frac{\partial}{\partial \xi_{i d}}+\frac{\partial}{\partial \xi_{(12)}}\right)\left(\xi_{(i d)}+\xi_{(12)}\right)=4=\frac{1}{d_{[1,1]}^{2}}\right. \\
\left\langle\tilde{\chi}_{[2],[2]} \mid \tilde{\chi}_{[1,1],[1,1]}\right\rangle & =4\left\langle\left\langle\tilde{\chi}_{[2]} \mid \tilde{\chi}_{[1,1]}\right\rangle=\frac{2^{2}}{2 !}\left(\frac{\partial}{\partial \xi_{i d}}-\frac{\partial}{\partial \xi_{(12)}}\right)\left(\xi_{i d}+\xi_{(12)}\right)=0\right. \\
\left\langle\tilde{\chi}_{[1,1],[1,1]} \mid \tilde{\chi}_{[2],[2]}\right\rangle & =4\left\langle\left\langle\tilde{\chi}_{[1,1]} \mid \tilde{\chi}_{[2]}\right\rangle=\frac{2^{2}}{2 !}\left(\frac{\partial}{\partial \xi_{i d}}+\frac{\partial}{\partial \xi_{(12)}}\right)\left(\xi_{i d}-\xi_{(12)}\right)=0\right. \\
\left\langle\tilde{\chi}_{[2],[2]} \mid \tilde{\chi}_{[2],[2]}\right\rangle & =4\left\langle\tilde{\chi}_{[2]} \mid \tilde{\chi}_{[2]}\right\rangle=\frac{2^{2}}{2 !}\left(\frac{\partial}{\partial \xi_{i d}}-\frac{\partial}{\partial \xi_{(12)}}\right)\left(\xi_{i d}-\xi_{(12)}\right)=4=\frac{1}{d_{[2]}^{2}}
\end{aligned}
$$

The difference between permutations and their conjugation classes first shows up at level $n=3$ :

$$
\begin{gathered}
\tilde{\chi}_{[3],[3]} \stackrel{(3.12)}{=} \frac{6}{3 !}\left(\mathcal{K}_{i d, i d}+\mathcal{K}_{i d,(12)}+\mathcal{K}_{i d,(13)}+\mathcal{K}_{i d,(23)}+\mathcal{K}_{i d,(123)}+\mathcal{K}_{i d,(132)}\right)= \\
=P_{1}^{3}+3 P_{2} P_{1}+2 P_{3}=6 \tilde{\chi}_{[3]} \\
\stackrel{(4.11)}{=} 6\left(\xi_{i d}+\xi_{(12)}+\xi_{(13)}+\xi_{(23)}+\xi_{(123)}+\xi_{(132)}\right) \\
\tilde{\chi}_{[2,1],[2,1]} \stackrel{(3.12)}{=} \frac{3}{3 !}\left(2 \mathcal{K}_{i d, i d}-\mathcal{K}_{i d,(123)}-\mathcal{K}_{i d,(132)}\right)=\frac{1}{2}\left(2 P_{1}^{3}-2 P_{3}\right)=3 \tilde{\chi}_{[2,1]} \\
\stackrel{(4.11)}{=} 3\left(2 \xi_{i d}-\xi_{(123)}-\xi_{(132)}\right) \\
\tilde{\chi}_{[1,1,1],[1,1,1]} \stackrel{(3.12)}{=} \frac{6}{3 !}\left(\mathcal{K}_{i d, i d}-\mathcal{K}_{i d,(12)}-\mathcal{K}_{i d,(13)}-\mathcal{K}_{i d,(23)}+\mathcal{K}_{i d,(123)}+\mathcal{K}_{i d,(132)}\right)= \\
=P_{1}^{3}-3 P_{2} P_{1}+2 P_{3}=6 \tilde{\chi}_{[1,1,1]} \\
\stackrel{(4.11)}{=} 6\left(\xi_{i d}-\xi_{(12)}-\xi_{(13)}-\xi_{(23)}+\xi_{(123)}+\xi_{(132)}\right)
\end{gathered}
$$

so that

$$
\begin{aligned}
& \left\langle\tilde{\chi}_{[1,1,1],[1,1,1]} \mid \tilde{\chi}_{[1,1,1],[1,1,1]}\right\rangle=6^{2}\left\langle\tilde{\chi}_{[1,1,1]} \mid \tilde{\chi}_{[1,1,1]}\right\rangle= \\
& =\frac{6^{2}}{3 !}\left(\frac{\partial}{\partial \xi_{i d}}+\frac{\partial}{\partial \xi_{(12)}}+\frac{\partial}{\partial \xi_{(13)}}+\frac{\partial}{\partial \xi_{(23)}}+\frac{\partial}{\partial \xi_{(123)}}+\frac{\partial}{\partial \xi_{(132)}}\right)\left(\xi_{i d}+\xi_{(12)}+\xi_{(13)}+\xi_{(23)}+\xi_{(123)}+\xi_{(132)}\right) \\
& =36=\frac{1}{d_{[1,1,1]}^{2}}\left\langle\tilde{\chi}_{[2,1],[2,1]} \mid \tilde{\chi}_{[2,1],[2,1]}\right\rangle \\
& =3^{2}\left\langle\tilde{\chi}_{[2,1]} \mid \tilde{\chi}_{[2,1]}\right\rangle=\frac{3^{2}}{3 !}\left(2 \frac{\partial}{\partial \xi_{i d}}-\frac{\partial}{\partial \xi_{(123)}}-\frac{\partial}{\partial \xi_{(132)}}\right)\left(2 \xi_{i d}-\xi_{(123)}-\xi_{(132)}\right)=9=\frac{1}{d_{[2,1]}^{2}}
\end{aligned}
$$

and so on. 
To conclude this section, we emphasize once again that $\tilde{\chi}$ are just linear functions of $\xi$-variables, and conjugate to $\xi$ are just $\xi$-derivatives, with nothing like factors $k$ in the conventional formalism with $p_{k}$-derivatives.

\subsection{Aristotelian model, $r=3$}

Now we consider the case of the Aristotelian model. In this case the character depends not only on the sums over the conjugation classes, hence, we need the full set of $\xi$-variables. Thus, the generalized character is

$$
\tilde{\chi}_{R_{1} R_{2} R_{3}}:=\frac{1}{n !} \sum_{\left\{\sigma_{i}\right\} \in S_{n}} \psi_{R_{1}}\left(\sigma_{1}\right) \psi_{R_{2}}\left(\sigma_{2}\right) \psi_{R_{3}}\left(\sigma_{3}\right) \cdot \eta_{\sigma_{1} \sigma_{2} \sigma_{3}}
$$

where $\eta_{\sigma_{1} \sigma_{2} \sigma_{3}}$ are indeterminants with the property $\eta_{\sigma_{1} \sigma_{2} \sigma_{3}}=\eta_{i d, \sigma_{1}^{-1} \circ \sigma_{2}, \sigma_{1}^{-1} \circ \sigma_{3}}$. Hence, similarly to (3.18), one can consider

$$
\tilde{\chi}_{R_{1} R_{2} R_{3}}:=\sum_{\gamma, \sigma_{1}, \sigma_{2} \in S_{n}} \psi_{R_{1}}(\gamma) \psi_{R_{2}}\left(\gamma \circ \sigma_{1}\right) \psi_{R_{3}}\left(\gamma \circ \sigma_{2}\right) \cdot \xi_{\sigma_{1} \sigma_{2}}
$$

with free ${ }^{2}$ indeterminants $\xi_{\sigma_{1} \sigma_{2}}$. Then, if we require that

$$
\left\langle\left\langle\xi_{\sigma_{1} \sigma_{2}} \mid \xi_{\sigma_{1}^{\prime} \sigma_{2}^{\prime}}\right\rangle=\frac{\delta_{\sigma_{1} \sigma_{1}^{\prime}} \delta_{\sigma_{2} \sigma_{2}^{\prime}}}{n !}\right.
$$

and apply (3.1), we get:

$$
\begin{aligned}
& \left\langle\tilde{\chi}_{R_{1} R_{2} R_{3}} \mid \tilde{\chi}_{R_{1}^{\prime} R_{2}^{\prime} R_{3}^{\prime}}\right\rangle \\
& =\frac{1}{n !} \sum_{\gamma, \gamma^{\prime}, \sigma_{1}, \sigma_{2} \in S_{n}} \psi_{R_{1}}(\gamma) \psi_{R_{2}}\left(\gamma \circ \sigma_{1}\right) \psi_{R_{3}}\left(\gamma \circ \sigma_{2}\right) \psi_{R_{1}^{\prime}}\left(\gamma^{\prime}\right) \psi_{R_{2}^{\prime}}\left(\gamma^{\prime} \circ \sigma_{1}\right) \psi_{R_{3}^{\prime}}\left(\gamma^{\prime} \circ \sigma_{2}\right)= \\
& =\frac{\delta_{R_{2} R_{2}^{\prime}} \delta_{R_{3} R_{3}^{\prime}}}{d_{R_{2}} d_{R_{3}}} \cdot \frac{1}{n !} \sum_{\gamma, \gamma^{\prime} \in S_{n}} \psi_{R_{1}}(\gamma) \psi_{R_{2}}\left(\gamma^{\prime} \circ \gamma^{-1}\right) \psi_{R_{3}}\left(\gamma^{\prime} \circ \gamma^{-1}\right) \psi_{R_{1}^{\prime}}\left(\gamma^{\prime}\right) \stackrel{\gamma^{\prime} \rightarrow \gamma^{\prime} \circ \gamma}{=} \\
& =\frac{\delta_{R_{2} R_{2}^{\prime}} \delta_{R_{3} R_{3}^{\prime}}}{d_{R_{2}} d_{R_{3}}} \cdot \frac{1}{n !} \sum_{\gamma, \gamma^{\prime} \in S_{n}} \psi_{R_{1}}(\gamma) \psi_{R_{2}}\left(\gamma^{\prime}\right) \psi_{R_{3}}\left(\gamma^{\prime}\right) \psi_{R_{1}^{\prime}}\left(\gamma^{\prime} \circ \gamma\right) \stackrel{(3.1)}{=} \\
& =\frac{\delta_{R_{1} R_{1}^{\prime}} \delta_{R_{2} R_{2}^{\prime}} \delta_{R_{3} R_{3}^{\prime}}}{d_{R_{1}} d_{R_{2}} d_{R_{3}}} \cdot \frac{1}{n !} \sum_{\gamma^{\prime} \in S_{n}} \psi_{R_{1}}\left(\gamma^{\prime}\right) \psi_{R_{2}}\left(\gamma^{\prime}\right) \psi_{R_{3}}\left(\gamma^{\prime}\right)=\frac{C_{R_{1} R_{2} R_{3}}}{d_{R_{1}} d_{R_{2}} d_{R_{3}}} \delta_{R_{1} R_{1}^{\prime}} \delta_{R_{2} R_{2}^{\prime}} \delta_{R_{3} R_{3}^{\prime}}
\end{aligned}
$$

where we used the definition of $C$ in (3.21). Finally, with the definitions (4.18) and (4.19),

$$
\left\langle\tilde{\chi}_{R_{1} R_{2} R_{3}} \mid \tilde{\chi}_{R_{1}^{\prime} R_{2}^{\prime} R_{3}^{\prime}}\right\rangle=\frac{C_{R_{1} R_{2} R_{3}}}{d_{R_{1}} d_{R_{2}} d_{R_{3}}} \delta_{R_{1} R_{1}^{\prime}} \delta_{R_{2} R_{2}^{\prime}} \delta_{R_{3} R_{3}^{\prime}}
$$

Comparing with (3.17) we see that $\eta$ in (4.17) and thus $\xi$ in (4.18) just substitute $\mathcal{K}$, while (4.19) defines a scalar product in the space of the relevant operators $\mathcal{K}$, which makes our $\chi_{\vec{R}}$ orthogonal.

\footnotetext{
${ }^{2}$ This is an essential point: we use more variables than necessary, there is an additional remaining symmetry $\xi_{\sigma_{1} \sigma_{2}}=\xi_{\gamma^{-1} \circ \sigma_{1} \circ \gamma, \gamma^{-1} \circ \sigma_{2} \circ \gamma}$, see [12] (in terms of that paper, we are working here in the RGgauge). This means that the generalized characters effectively depend on less variables: they depend only on some combinations of $\xi_{\sigma_{1} \sigma_{2}}$. They can be chosen, for instance, as sums over conjugacy classes of $\sigma_{2}$, similarly to what we did in the case of $r=2$. However, we ignore this subtlety here.
} 


\subsection{Other rainbow models, arbitrary $r$}

In complete analogy with the $r=3$ case, one can consider an arbitrary $r$. Then we define

$$
\tilde{\chi}_{\vec{R}}:=\sum_{\gamma,\left\{\sigma_{j}\right\} \in S_{n}} \psi_{R_{1}}(\gamma) \prod_{j=1}^{r-1} \psi_{R_{j+1}}\left(\gamma \circ \sigma_{j}\right) \cdot \xi_{\vec{\sigma}}
$$

require that

$$
\left\langle\xi_{\vec{\sigma}} \mid \xi_{\vec{\sigma}^{\prime}}\right\rangle=\frac{1}{n !} \prod_{i=1}^{r-1} \delta_{\sigma_{i} \sigma_{i}^{\prime}}
$$

and obtain

$$
\left\langle\left\langle\tilde{\chi}_{\vec{R}} \mid \tilde{\chi}_{\vec{R}^{\prime}}\right\rangle=\frac{C_{\vec{R}}}{\prod_{i=1}^{r} d_{R_{i}}} \prod_{i=1}^{r} \delta_{R_{i} R_{i}^{\prime}}\right.
$$

Despite somewhat formal introduction of linear $\xi$-variables and associated scalar product, this allows us to make the next step: to introduce generalized cut-and-join operators $\hat{W}$, for which our $\chi_{\vec{R}}$ are the common eigenfunctions.

\subsection{Generalized Cauchy formula}

A central role in applications of character theory is played by a rather elementary Cauchy formula for the bilinear sum of characters, see [50] for a brief survey. It is therefore important that it has a direct counterpart for the multilinear sum related to the tensorial characters:

$$
\begin{aligned}
\sum_{R_{1}, \ldots, R_{r}} C_{R_{1} \ldots R_{r}} \prod_{m=1}^{r} \chi_{R_{m}}\left\{p^{(m)}\right\} & \stackrel{(3.33)}{=} \sum_{\Delta} \frac{1}{z_{\Delta}} \prod_{m=1}^{r}\left(\sum_{R_{m}} \psi_{R_{m}}(\Delta) \chi_{R_{m}}\left\{p^{(m)}\right\}\right)= \\
& =\sum_{\Delta} \frac{1}{z_{\Delta}} \prod_{m=1}^{r} p_{\Delta}^{(m)}=\exp \left(\sum_{k} \frac{\prod_{m=1}^{r} p_{k}^{(m)}}{k}\right)
\end{aligned}
$$

where we used

$$
\sum_{R} \psi_{R}(\Delta) \chi_{R}\{p\}=p_{\Delta}
$$

which follows from the orthogonality condition

$$
\sum_{R} \psi_{R}(\Delta) \psi_{R}\left(\Delta^{\prime}\right)=z_{\Delta} \delta_{R, R^{\prime}}
$$

and also used the fact that

$$
\sum_{\Delta} \frac{p_{\Delta}}{z_{\Delta}}=\sum_{n} \chi_{[n]}\{p\}=\exp \left(\sum_{k} \frac{p_{k}}{k}\right)
$$

the last equality being nothing but the ordinary Cauchy formula. 
Partition function is a rather straightforward deformation of the l.h.s. of (4.25), which provides a tensorial generalization of $\mathrm{KP} /$ Toda tau-function, which we describe elsewhere [51]:

$$
\begin{aligned}
Z_{r}\left\{p^{(i)}\right\} & :=\sum_{R_{1}, \ldots, R_{r}} \prod_{j} \chi_{R_{j}}\left\{p^{(j)}\right\} \cdot\left\langle\chi_{R_{1}, \ldots, R_{r}}\right\rangle \\
& \stackrel{(1.5)}{=} \sum_{R_{1}, \ldots, R_{r}} C_{R_{1} \ldots R_{r}} \prod_{m=1}^{r}\left(\chi_{R_{m}}\left\{p^{(m)}\right\} \cdot \prod_{(i, j) \in R_{m}}\left(N_{m}+i-j\right)\right)
\end{aligned}
$$

\section{$5 \hat{W}$-operators}

\subsection{Cut-and-join operators of $[52,53]$}

For the usual Schur characters, one can construct a system of commuting differential operators $\hat{W}_{\Delta}$ that have these characters as a common system of their eigenfunctions, the eigenvalue of these operators being $[52,53]$

$$
\hat{W}_{\Delta} \chi_{R}=\frac{\psi_{R}(\Delta)}{d_{R}} \cdot \chi_{R}
$$

The operators are called generalized cut and join operator [52, 53], and one can construct them in terms of invariant matrix derivatives:

$$
\hat{W}_{\Delta}=: \prod_{i} \hat{D}_{\delta_{i}}:
$$

and

$$
\hat{D}_{k}=\operatorname{Tr}\left(M \partial_{M}\right)^{k}
$$

where $M$ is a matrix. The normal ordering in (5.2) implies that all the derivatives $\partial_{M}$ stand to the right of all $M$. Since $W_{\Delta}$ are "gauge"-invariant matrix operators, and we apply them only to gauge invariants, they can be realized as differential operators in the time-variables $P_{k}=\operatorname{Tr} M^{k}[52,53]$. In particular, the simplest cut-and-join operator $\hat{W}_{[2]},[54]$ is

$$
\hat{W}_{[2]}=\frac{1}{2} \sum_{a, b}\left((a+b) P_{a} P_{b} \partial_{a+b}+a b P_{a+b} \partial_{a} \partial_{b}\right)
$$

These operators act on the characters considered as functions of times $p_{k}$ so that (5.1) reads as

$$
\hat{W}_{\Delta} \chi_{R}\left\{p_{k}\right\}=\frac{\psi_{R}(\Delta)}{d_{R}} \cdot \chi_{R}\left\{p_{k}\right\}
$$

Note that this formula can be extended to the case of $|\Delta| \neq|R|$. In this case, the formula looks like

$$
\hat{W}_{\Delta} \chi_{R}\left(p_{k}\right)=\left\{\begin{array}{cl}
0 & \text { for }|\Delta|>|R| \\
C_{|R|-|\Delta|+r}^{r} \frac{1}{d_{R}} \psi_{R}([\Delta, \underbrace{1 \ldots 1}_{|R|-|\Delta|}]) \cdot \chi_{R}\left(p_{k}\right) & \text { for }|\Delta| \leq|R|
\end{array}\right.
$$

where $C_{n}^{k}:=\frac{n !}{(n-k) ! k !}$ are the binomial coefficients, and $r$ is the number of unit length cycles in the Young diagram $\Delta$. 


\section{2 $\hat{W}$-operators in rainbow tensor models}

As a direct generalization of (5.2), one can associate a $\hat{W}$-operator with any $\mathcal{K}_{\vec{\sigma}}$ :

$$
\hat{\mathcal{W}}_{\vec{\sigma}}:=: \mathcal{K}_{\vec{\sigma}}\left(\bar{M} \longrightarrow \frac{\partial}{\partial M}\right):
$$

where normal ordering means that all $M$-derivatives stand to the right of all $M$. Then, in the abbreviated notation of (3.31),

$$
\hat{\mathcal{W}}_{\vec{\sigma}} \mathcal{K}_{\vec{\sigma}^{\prime}}=\prod_{p=1}^{n} M_{\vec{a}_{p}} \frac{\partial}{\partial M_{\vec{a}_{\vec{\sigma}(p)}}} \prod_{p=1}^{n} M_{\vec{a}_{p}^{\prime}} \bar{M}_{\vec{a}_{\vec{\sigma}^{\prime}(p)}^{\prime}}=\sum_{\gamma \in S_{n}} \mathcal{K}_{\vec{\sigma} \circ \vec{\sigma}^{\prime} \circ \gamma}
$$

because $M$ derivatives are non-zero if the set of indices $\left\{\vec{a}_{\vec{\sigma}(p)}\right\}$ coincides with the set $\left\{a_{p}^{\prime}\right\}$, i.e. the indices themselves are equal modulo some $k$-independent permutation $\gamma \in S_{n}$, i.e. $a_{\sigma_{k}(p)}^{(k)}=a_{\left.\gamma_{(}\right)}^{\prime(k)}$. In the simplest example of $r=1$ and $n=2$,

$$
\begin{aligned}
\mathcal{W}_{\sigma} \mathcal{K}_{\sigma^{\prime}} & =\sum_{a, b=1}^{N} M_{a} M_{b} \frac{\partial^{2}}{\partial M_{\sigma(a)} \partial M_{\sigma_{b}}} \sum_{c, d=1}^{N} M_{c} M_{d} \bar{M}^{\sigma^{\prime}(c)} \bar{M}^{\sigma^{\prime}(d)}= \\
& =\sum_{a, b, c, d=1}^{N}\left(\delta_{c}^{\sigma(a)} \delta_{d}^{\sigma(b)}+\delta_{c}^{\sigma(b)} \delta_{d}^{\sigma(a)}\right) \cdot M_{a} M_{b} \bar{M}^{\sigma^{\prime}(c)} \bar{M}^{\sigma^{\prime}(d)}= \\
& =\sum_{a, b=1}^{N} M_{a} M_{b}\left(\bar{M}^{\sigma^{\prime} \circ \sigma(a)} \bar{M}^{\sigma^{\prime} \circ \sigma(b)}+\bar{M}^{\sigma^{\prime} \circ \sigma(b)} \bar{M}^{\sigma^{\prime} \circ \sigma(a)}\right)= \\
& =\sum_{\gamma \in S_{2}} M_{a} M_{b} \bar{M}^{\sigma^{\prime} \circ \sigma \circ \gamma(a)} \bar{M}^{\sigma^{\prime} \circ \sigma \circ \gamma(b)}=\sum_{\gamma \in S_{2}} \mathcal{K}_{\sigma^{\prime} \circ \sigma \circ \gamma}
\end{aligned}
$$

In fact, $\mathcal{K}$ and thus $\hat{\mathcal{W}}$ are invariant w.r.t. the common multiplication of all $n$ permutations $\sigma_{k}$ by a common $\sigma$, what allows to eliminate one of them,

$$
\mathcal{K}_{\vec{\sigma}}=\mathcal{K}_{\sigma_{1}, \ldots, \sigma_{r}}=\mathcal{K}_{\sigma \circ \sigma_{1}, \ldots, \sigma \circ \sigma_{r}}=\mathcal{K}_{i d, \sigma_{1}^{-1} \circ \sigma_{2}, \ldots, \sigma_{1}^{-1} \circ \sigma_{r}}
$$

moreover, there is still a freedom in common conjugation of the remaining $r-1$ permutations:

$$
\mathcal{K}_{i d, \sigma_{2}, \ldots, \sigma_{r}}=\mathcal{K}_{i d, \gamma \circ \sigma_{2} \gamma^{-1}, \ldots, \gamma \circ \sigma_{r} \gamma^{-1}}
$$

In the case of RCM $(r=2)$, this means that $\mathcal{K}$ depend only on the conjugation class of $\sigma_{1}^{-1} \circ \sigma_{2}$, i.e. on a Young diagram, but for $r>2$ the classification is more complicated, see [12] for details.

For our present purposes, we just need to eliminate $\sigma_{1}$ and $\sigma_{1}^{\prime}$ from (5.8). For example, for $r=2$

$$
\begin{aligned}
\hat{\mathcal{W}}_{i d, \sigma} \mathcal{K}_{i d, \sigma^{\prime}} & =\sum_{\vec{a}, \vec{b}, \vec{c}, \vec{d}} M_{a_{1} b_{1}} \ldots M_{a_{n} b_{n}} \frac{\partial^{n}}{\partial M_{a_{1} b_{\sigma(1)}} \ldots \partial M_{a_{n} b_{\sigma(n)}}} M_{c_{1} d_{1}} \ldots M_{c_{n} d_{n}} \bar{M}^{c_{1} d_{\sigma^{\prime}(1)}} \ldots \bar{M}^{c_{n} d_{\sigma^{\prime}(n)}}= \\
& =\sum_{\vec{a}, \vec{b}, \vec{c}, \vec{d}} M_{a_{1} b_{1}} \ldots M_{a_{n} b_{n}} \bar{M}^{c_{1} d_{\sigma^{\prime}(1)}} \ldots \bar{M}^{c_{n} d_{\sigma^{\prime}(n)}} \sum_{\gamma \in S_{n}} \prod_{p=1}^{n} \delta_{c_{p}}^{a_{\gamma(p)}} \delta_{d_{p}}^{b_{\sigma \sigma \gamma(p)}}=\sum_{\gamma \in S_{n}} \mathcal{K}_{\gamma, \sigma^{\prime} \circ \sigma \circ \gamma}= \\
& =\sum_{\gamma \in S_{n}} \mathcal{K}_{i d, \gamma^{-1} \circ \sigma^{\prime} \circ \sigma \circ \gamma}=n ! \cdot \mathcal{K}_{i d, \sigma^{\prime} \circ \sigma}
\end{aligned}
$$


This remains true for arbitrary $r$ :

$$
(5.8) \Longrightarrow \hat{\mathcal{W}}_{i d, \vec{\sigma}} \mathcal{K}_{i d, \vec{\sigma}^{\prime}}=n ! \cdot \mathcal{K}_{i d, \vec{\sigma} \circ \vec{\sigma}^{\prime}}
$$

where now $\vec{\sigma}$ denotes a set of $r-1$ permutations from $S_{n}$.

We can now apply the $\hat{\mathcal{W}}$-operator to our $\chi$ :

$$
\begin{aligned}
\hat{\mathcal{W}}_{\vec{\sigma}} \chi_{\vec{R}}=\frac{1}{n !} \sum_{\vec{\sigma} \in S_{n}} \Psi_{\vec{R}}\left(\vec{\sigma}^{\prime}\right) \hat{W}_{\vec{\sigma}} \mathcal{K}_{\vec{\sigma}^{\prime}} & =\frac{1}{n !} \sum_{\gamma, \vec{\sigma}^{\prime} \in S_{n}} \Psi_{\vec{R}}\left(\vec{\sigma}^{\prime}\right) \mathcal{K}_{\vec{\sigma} \circ \vec{\sigma}^{\prime} \circ \gamma}= \\
& =\frac{1}{n !} \sum_{\gamma, \vec{\sigma}^{\prime} \in S_{n}} \Psi_{\vec{R}}\left(\vec{\sigma}^{-1} \circ \vec{\sigma} \circ \gamma^{-1}\right) \mathcal{K}_{\vec{\sigma}^{\prime}}
\end{aligned}
$$

where $\Psi_{\vec{R}}(\vec{\sigma}):=\prod_{p=1}^{n} \psi_{R_{i}}\left(\sigma_{i}\right)$. This expression can be simplified a little, at expense of breaking explicit $S_{n}$ symmetry, by using (5.12) instead of (5.8) and (3.18) instead of (3.17):

$$
\begin{aligned}
& \hat{\mathcal{W}}_{i d, \sigma_{2}, \ldots, \sigma_{r}} \chi_{R_{1}, \ldots, R_{r}}=\frac{1}{n !} \sum_{\sigma_{1}^{\prime}, \ldots, \sigma_{r}^{\prime} \in S_{n}} \psi_{R_{1}}\left(\sigma_{1}^{\prime}\right) \ldots \psi_{R_{r}}\left(\sigma_{r}^{\prime}\right) \hat{\mathcal{W}}_{i d, \sigma_{2}, \ldots, \sigma_{r}} \mathcal{K}_{\sigma_{1}^{\prime}, \sigma_{2}^{\prime}, \ldots, \sigma_{r}^{\prime}}= \\
& =\frac{1}{n !} \sum_{\sigma_{1}^{\prime}, \ldots, \sigma_{r}^{\prime} \in S_{n}} \psi_{R_{1}}\left(\sigma_{1}^{\prime}\right) \psi_{R_{2}}\left(\sigma_{1}^{\prime} \circ \sigma_{2}^{\prime}\right) \ldots \psi_{R_{r}}\left(\sigma_{1}^{\prime} \circ \sigma_{r}^{\prime}\right) \hat{\mathcal{W}}_{i d, \sigma_{2}, \ldots, \sigma_{r}} \mathcal{K}_{i d, \sigma_{2}^{\prime}, \ldots, \sigma_{r}^{\prime}}= \\
& \stackrel{(5.12)}{=} \sum_{\sigma_{1}^{\prime}, \ldots, \sigma_{r}^{\prime} \in S_{n}} \psi_{R_{1}}\left(\sigma_{1}^{\prime}\right) \psi_{R_{2}}\left(\sigma_{1}^{\prime} \circ \sigma_{2}^{\prime}\right) \ldots \psi_{R_{r}}\left(\sigma_{1}^{\prime} \circ \sigma_{r}^{\prime}\right) \mathcal{K}_{i d, \sigma_{2} \circ \sigma_{2}^{\prime}, \ldots, \sigma_{r} \circ \sigma_{r}^{\prime}}
\end{aligned}
$$

\subsection{RCM, $r=2$}

In this case, we get just

$$
\begin{aligned}
\hat{\mathcal{W}}_{i d, \sigma} \chi_{R_{1}, R_{2}} & =\sum_{\sigma_{1}^{\prime}, \sigma_{2}^{\prime} \in S_{n}} \psi_{R_{1}}\left(\sigma_{1}^{\prime}\right) \psi_{R_{2}}\left(\sigma_{1}^{\prime} \circ \sigma_{2}^{\prime}\right) \mathcal{K}_{i d, \sigma \circ \sigma_{2}^{\prime}} \stackrel{(3.1)}{=} \frac{\delta_{R_{1}, R_{2}}}{d_{R_{1}}} \sum_{\sigma_{2}^{\prime}} \psi_{R_{1}}\left(\sigma_{2}^{\prime}\right) \mathcal{K}_{i d, \sigma \circ \sigma_{2}^{\prime}}= \\
& =\frac{\delta_{R_{1}, R_{2}}}{d_{R_{1}}} \sum_{\sigma_{2}^{\prime}} \psi_{R_{1}}\left(\sigma^{-1} \circ \sigma_{2}^{\prime}\right) \mathcal{K}_{i d, \sigma_{2}^{\prime}}= \\
& =\frac{\delta_{R_{1}, R_{2}}}{d_{R_{1}}} \cdot \frac{\psi_{R_{1}}(\sigma)}{\left|R_{1}\right| ! \cdot d_{R_{1}}} \sum_{\sigma_{2}^{\prime}} \psi_{R_{1}}\left(\sigma_{2}^{\prime}\right) \mathcal{K}_{i d, \sigma_{2}^{\prime}} \stackrel{(3.12)}{=} \frac{\psi_{R_{1}}(\sigma)}{d_{R_{1}}} \cdot \chi_{R_{1}, R_{2}}
\end{aligned}
$$

To check the next to the last transition, one can substitute $\mathcal{K}_{i d, \gamma}$ by arbitrary $\psi_{Q}(\gamma)$ (make a Fourier transform) and then use the orthogonality relations:

$$
\sum_{\gamma \in S_{n}} \psi_{R}\left(\sigma^{-1} \circ \gamma\right) \psi_{Q}(\gamma) \stackrel{(3.1)}{=} \frac{\psi_{R}\left(\sigma^{-1}\right)}{d_{R}} \delta_{Q R}=\frac{\psi_{R}(\sigma)}{d_{R}} \delta_{Q R} \stackrel{(3.3)}{=} \frac{\psi_{R}(\sigma)}{d_{R} \cdot|R| !} \sum_{\gamma} \psi_{R}(\gamma) \psi_{Q}(\gamma)
$$

For the validity of this trick, it is important that both $\mathcal{K}_{i d, \gamma}$ and $\psi_{Q}(\gamma)$ depend only on the conjugation class of $\gamma$, so that above transform is actually invertible. We used also the fact that $\sigma^{-1}$ and $\sigma$ belong to the same conjugation class, so that $\psi_{R}\left(\sigma^{-1}\right)=\psi_{R}(\sigma)$. 
Thus, we obtain that

$$
\hat{\mathcal{W}}_{\sigma} \chi_{R_{1} R_{2}}=\lambda_{R_{1} R_{2}}^{\sigma} \cdot \chi_{R_{1} R_{2}}
$$

where the eigenvalues are

$$
\lambda_{R_{1} R_{2}}^{\sigma}=\frac{\psi_{R_{1}}(\sigma)}{d_{R_{1}}}
$$

\subsection{Aristotelian model, $r=3$}

In the case of $r=3$ we have:

$$
\begin{aligned}
\hat{\mathcal{W}}_{\sigma_{1} \sigma_{2}} \chi_{R_{1} R_{2} R_{3}} \stackrel{(3.17)}{=} \frac{1}{n !} \sum_{\left\{\gamma_{1}, \gamma_{2}, \gamma_{3}\right\} \in S_{n}} \psi_{R_{1}}\left(\gamma_{1}\right) \psi_{R_{2}}\left(\gamma_{2}\right) \psi_{R_{3}}\left(\gamma_{3}\right) \hat{W}_{\sigma_{1} \sigma_{2}} \mathcal{K}_{\gamma_{1}, \gamma_{2}, \gamma_{3}}= \\
=\frac{1}{n !} \sum_{\left\{\gamma_{1}, \gamma_{2}, \gamma_{3}\right\} \in S_{n}} \psi_{R_{1}}\left(\gamma_{1}\right) \psi_{R_{2}}\left(\gamma_{2}\right) \psi_{R_{3}}\left(\gamma_{3}\right) \hat{W}_{\sigma_{1} \sigma_{2}} \mathcal{K}_{i d, \gamma_{1}^{-1} \circ \gamma_{2}, \gamma_{1}^{-1} \circ \gamma_{3}}= \\
=\frac{1}{n !} \sum_{\left\{\gamma_{1}, \gamma_{2}, \gamma_{3}\right\} \in S_{n}} \psi_{R_{1}}\left(\gamma_{1}\right) \psi_{R_{2}}\left(\gamma_{1} \circ \gamma_{2}\right) \psi_{R_{3}}\left(\gamma_{1} \circ \gamma_{3}\right) \hat{W}_{\sigma_{1} \sigma_{2}} \mathcal{K}_{i d, \gamma_{2}, \gamma_{3}}
\end{aligned}
$$

If sizes $\left|\sigma_{i}\right|$ are equal to $\left|R_{i}\right|$, then, as direct generalization of (5.11), the $\hat{W}$-operator acts as averaging over the permutation group $S_{n}$ :

$$
\hat{\mathcal{W}}_{\sigma_{1} \sigma_{2}} \mathcal{K}_{i d, \gamma_{2}, \gamma_{3}}=n ! \cdot \mathcal{K}_{i d, \gamma_{2} \circ \sigma_{1}, \gamma_{3} \circ \sigma_{2}}=\sum_{\gamma \in S_{n}} \mathcal{K}_{i d, \gamma \circ \gamma_{2} \circ \sigma_{1} \circ \gamma^{-1}, \gamma \circ \gamma_{3} \circ \sigma_{2} \circ \gamma^{-1}}
$$

In the last transition, we used invariance of operators $\mathcal{K}_{i d, \gamma_{2}, \gamma_{3}}$ under conjugation, see footnote 2 .

\section{$5.5 \hat{W}$-operators in $\xi$-variables}

At the last step in (5.11), we used the fact that the operators $\mathcal{K}_{i d, \gamma}$ are invariant w.r.t. the conjugation by arbitrary $\gamma^{\prime}$. However, we can ignore this symmetry and again consider the variables $\xi_{\sigma}$ without this additional invariance. In particular, the $\hat{W}$-operators can be realized as differential operators in these variables,

$$
\hat{W}_{\sigma}=\sum_{\gamma, \gamma^{\prime} \in S_{n}} \xi_{\gamma^{\prime} \circ \gamma \circ \sigma \circ \gamma^{\prime-1}} \frac{\partial}{\partial \xi_{\gamma}}
$$

Such generalized cut and join operators are linear in contrast with those in $p$-variables. As usual, formulas in $\xi$-variables are looking simpler, for the price of enlarging the space of variables. 


\subsubsection{The case of RCM, $r=2$}

We can re-deduce (5.15) in $\xi$-variables: from (4.11)

$$
\begin{aligned}
\hat{W}_{\sigma} \tilde{\chi}_{R} & =\sum_{\gamma, \gamma^{\prime} \in S_{n}} \xi_{\gamma^{\prime} \circ \gamma \circ \sigma \circ \gamma^{\prime-1}} \frac{\partial}{\partial \xi_{\gamma}} \sum_{\gamma \in S_{n}} \psi_{R}(\gamma) \cdot \xi_{\gamma}= \\
& =\sum_{\gamma, \gamma^{\prime} \in S_{n}} \psi_{R}(\gamma) \cdot \xi_{\gamma^{\prime} \circ \gamma \circ \sigma \circ \gamma^{\prime-1}}=\sum_{\gamma, \gamma^{\prime} \in S_{n}} \psi_{R}\left(\gamma \circ \sigma^{-1}\right) \cdot \xi_{\gamma^{\prime} \circ \gamma \circ \gamma^{\prime-1}}= \\
& =\frac{\psi_{R}(\sigma)}{d_{R} \cdot n !} \sum_{\gamma \gamma^{\prime} \in S_{n}} \psi_{R}(\gamma) \cdot \xi_{\gamma^{\prime} \circ \gamma \circ \gamma^{\prime-1}}=\frac{\psi_{R}(\sigma)}{d_{R} \cdot n !} \sum_{\gamma \gamma^{\prime} \in S_{n}} \psi_{R}\left(\gamma^{\prime-1} \circ \gamma \circ \gamma^{\prime}\right) \cdot \xi_{\gamma}= \\
& =\frac{\psi_{R}(\sigma)}{d_{R}} \sum_{\gamma \in S_{n}} \psi_{R}(\gamma) \cdot \xi_{\gamma}=\frac{\psi_{R}(\sigma)}{d_{R}} \cdot \tilde{\chi}_{R}
\end{aligned}
$$

The transition between the two lines can be explained just by the same trick: despite, in variance with $\mathcal{K}_{i d, \gamma}$, the variables $\xi_{\gamma}$ are not supposed to be invariant under conjugations, thus they could not be just substituted by invariant $\psi_{Q}(\gamma)$, the sums $\sum_{\gamma^{\prime} \in S_{n}} \xi_{\gamma^{\prime} \circ \gamma \circ \sigma \circ \gamma^{\prime-1}}$ are invariant and can be substituted so that the trick can be used.

If now one chooses $\sigma$ that labels $\hat{W}$-operators, and $R_{1}, R_{2}$ that label the character belonging to different symmetric groups, $S_{n}$ and $S_{m}$ correspondingly, the property (5.17) still persists. It is clear that, when $n>m, \lambda_{R_{1} R_{2}}^{\sigma}=0$. Otherwise, one has to extend permutations from $S_{n}$ to those from $S_{m}$ adding trivial cycles so that

$$
\lambda_{R_{1} R_{2}}^{\sigma}=\left\{\begin{array}{cc}
0 & \text { for } n>m \\
C_{m-n+p}^{p} \frac{\psi_{R_{1}}\left(\sigma()^{m-n}\right)}{d_{R_{1}}} & \text { for } n \leq m
\end{array}\right.
$$

where ()$^{k}$ means $k$ trivial cycles added to the permutation, and $p$ is the number of trivial cycles in the permutation $\sigma$. This is a counterpart of the original extension formula (5.6).

\subsubsection{Aristotelian model, $r=3$}

In this case,

$$
\hat{W}_{\sigma_{1} \sigma_{2}}=\sum_{\gamma, \gamma_{1} \gamma_{2} \in S_{n}} \xi_{\gamma \circ \gamma_{2} \circ \sigma_{1} \circ \gamma^{-1}, \gamma \circ \gamma_{3} \circ \sigma_{2} \circ \gamma^{-1}} \frac{\partial}{\partial \xi_{\gamma_{1} \gamma_{2}}}
$$

One can check for symmetric groups $S_{n}$ with small $n$ that, when all sizes $\left|\sigma_{i}\right|=\left|R_{i}\right|=n$,

$$
\hat{W}_{\sigma_{1} \sigma_{2}} \tilde{\chi}_{R_{1} R_{2} R_{3}}=\lambda_{R_{1} R_{2} R_{3}}^{\sigma_{1} \sigma_{2}} \cdot \tilde{\chi}_{R_{1} R_{2} R_{3}}
$$

where the eigenvalues are

$$
\bar{\lambda}_{R_{1} R_{2} R_{3}}^{\sigma_{1} \sigma_{2}} \frac{\sum_{\gamma \in S_{n}} \psi_{R_{1}}(\gamma) \psi_{R_{2}}\left(\gamma \circ \sigma_{1}\right) \psi_{R_{3}}\left(\gamma \circ \sigma_{2}\right)}{C_{R_{1} R_{2} R_{3}}}=\frac{C_{R_{1} R_{2} R_{3}}^{\sigma_{1} \sigma_{2}}}{C_{R_{1} R_{2} R_{3}}} \quad \text { at } C_{R_{1} R_{2} R_{3}} \neq 0
$$

since otherwise, when $C_{R_{1} R_{2} R_{3}}=0, \tilde{\chi}_{R_{1} R_{2} R_{3}}=0$. Now one could try to repeat the trick that was used in the proof of (3.4) and try to prove (5.24), i.e. that

$C_{R_{1} R_{2} R_{3}} \sum_{\gamma, \gamma^{\prime}} \psi_{R_{1}}\left(\gamma^{\prime}\right) \psi_{R_{2}}\left(\gamma^{\prime} \circ \gamma_{1} \circ \gamma \circ \sigma_{1} \circ \gamma^{-1}\right) \psi_{R_{3}}\left(\gamma^{\prime} \circ \gamma_{2} \circ \gamma \circ \sigma_{2} \circ \gamma^{-1}\right)=C_{R_{1} R_{2} R_{3}}^{\sigma_{1} \sigma_{2}} C_{R_{1} R_{2} R_{3}}^{\gamma_{1} \gamma_{2}}$ 
Indeed, one can make a "Fourier" transform of this formula with the kernel $C_{Q_{1} Q_{2} Q_{3}}^{\sigma_{1} \sigma_{2}}$ which gives for its l.h.s.

$$
\begin{aligned}
& C_{R_{1} R_{2} R_{3}} \sum_{\sigma_{1} \sigma_{2}} C_{Q_{1} Q_{2} Q_{3}}^{\sigma_{1} \sigma_{2}} \sum_{\gamma, \gamma^{\prime}} \psi_{R_{1}}\left(\gamma^{\prime}\right) \psi_{R_{2}}\left(\gamma^{\prime} \circ \gamma_{1} \circ \gamma \circ \sigma_{1} \circ \gamma^{-1}\right) \psi_{R_{3}}\left(\gamma^{\prime} \circ \gamma_{2} \circ \gamma \circ \sigma_{2} \circ \gamma^{-1}\right)= \\
& =C_{R_{1} R_{2} R_{3}} \frac{C_{Q_{1} Q_{2} Q_{3}}^{\gamma_{1} \gamma_{2}}}{d_{R_{1}} d_{R_{2}} d_{R_{3}}} \delta_{R_{1} Q_{1}} \delta_{R_{2} Q_{2}} \delta_{R_{3} Q_{3}}
\end{aligned}
$$

and the same for the r.h.s.:

$$
\sum_{\sigma_{1} \sigma_{2}} C_{Q_{1} Q_{2} Q_{3}}^{\sigma_{1} \sigma_{2}} C_{R_{1} R_{2} R_{3}}^{\sigma_{1} \sigma_{2}} C_{R_{1} R_{2} R_{3}}^{\gamma_{1} \gamma_{2}}=\frac{C_{Q_{1} Q_{2} Q_{3}}^{\gamma_{1} \gamma_{2}} C_{R_{1} R_{2} R_{3}}}{d_{R_{1}} d_{R_{2}} d_{R_{3}}} \delta_{R_{1} Q_{1}} \delta_{R_{2} Q_{2}} \delta_{R_{3} Q_{3}}
$$

Thus, these two formulas coincide. Unfortunately, this does not prove (5.26), since, though the 1.h.s. of (5.26) does not change upon simultaneous conjugation of $\sigma_{1}$ and $\sigma_{2}$ (and, similarly, $\gamma_{1}$ and $\gamma_{2}$ ), the Fourier transform has no non-trivial kernel only for symmetric groups $S_{n}$ with small enough $n<5 .{ }^{3}$ Hence, (5.26) is proved only for these groups. We, however, have checked with the computer that this formula is correct for various concrete cases in $S_{5}$ and $S_{6}$.

In this $r=3$ case, one again can choose $\sigma_{i}$ labelling $\hat{W}$, and $R_{i}$ labelling the generalized character belonging to different symmetric groups, $S_{n}$ and $S_{m}$ correspondingly. In this case, the property (5.24) still persists. It is clear that, when $n>m, \lambda_{R_{1} R_{2} R_{3}}^{\sigma_{1} \sigma_{2}}=0$. Otherwise, one has to extend permutations from $S_{n}$ to those from $S_{m}$ adding trivial cycles, so that

$$
\lambda_{R_{1} R_{2} R_{3}}^{\sigma_{1} \sigma_{2}}=\left\{\begin{array}{cc}
0 & \text { for } n>m \\
C_{m-n+p}^{p} \bar{\lambda}_{R_{1} R_{2} R_{3}}^{\sigma_{1}()^{m-n}, \sigma_{2}()^{m-n}} & \text { for } n \leq m
\end{array}\right.
$$

where $p$ is the number of points left intact under the action of both permutations $\sigma_{1}$ and $\sigma_{2}$. This is an Aristotelian counterpart of (5.6) and (5.22).

\subsubsection{Generic $r$}

Similarly, in general, when $\left|\sigma_{i}\right|=\left|R_{i}\right|=n$, one can also make a statement, which is proved only for small symmetric groups, that

$$
\hat{W}_{\vec{\sigma}} \tilde{\chi}_{\vec{R}}=\lambda_{\vec{R}}^{\vec{\sigma}} \cdot \tilde{\chi}_{\vec{R}}
$$

where $\vec{R}$ is a set of representations $R_{1}, \ldots, R_{r}$ and the eigenvalues are

$$
\begin{aligned}
\bar{\lambda}_{\vec{R}}^{\vec{\sigma}} & =\frac{\sum_{\gamma \in S_{n}} \psi_{R_{1}}(\gamma) \prod_{j=1}^{r-1} \psi_{R_{j+1}}\left(\gamma \circ \sigma_{j}\right)}{C_{R_{1}, \ldots, R_{r}}} \\
& =n ! \cdot \frac{\sum_{\gamma \in S_{n}} \psi_{R_{1}}(\gamma) \prod_{j=1}^{r-1} \psi_{R_{j+1}}\left(\gamma \circ \sigma_{j}\right)}{\sum_{\gamma \in S_{n}} \prod_{j=1}^{r} \psi_{R_{j}}(\gamma)} \text { at } C_{R_{1}, \ldots, R_{r}} \neq 0
\end{aligned}
$$

\footnotetext{
${ }^{3}$ Existence of a non-trivial kernel for large enough $n$ is clear already from the fact that dimension of the space of pairs $\left(\sigma_{1}, \sigma_{2}\right)$ invariant w.r.t. the common conjugation [12], $D=\sum_{\Delta \vdash n} z_{\Delta}$ grows factorially with $n$, while the number of ordered triples of Young diagrams, much slower, (see [12], s.6.1.2).
} 
In the generic case of $|\Delta|=n$ not equal to $|R|=m$, as the generalization of (5.6) and (5.22),

$$
\lambda_{\vec{R}}^{\vec{\sigma}}=\left\{\begin{array}{cc}
0 & \text { for } n>m \\
C_{m-n+p}^{p} \bar{\lambda}_{\vec{R}}^{\vec{\sigma}()^{m-n}} & \text { for } n \leq m
\end{array}\right.
$$

where $p$ is the number of points left intact under the action of all the permutations $\sigma_{i}$.

\section{Conclusion}

In this paper, we focused on the property (1.1), which is extremely well suited to gaining our knowledge in the tensor case, and thus provides a solid base for bringing new progress. Namely, the averages in the Aristotelian model of [12] are some polynomials in $N$ 's, with the properties

(i) they are not generic;

(ii) they are tri-linear in dimensions;

(iii) at a given level, only some of the tri-linear products appear;

(iv) these tri-linear combinations are averages of linear combinations of $\mathcal{K}$-operators with appropriate symmetries;

(v) combinations of $\mathcal{K}$ 's with the symmetries which do not appear in the list of tri-linear combinations identically vanish: this is the reason why they do not appear among averages;

(vi) all this remains true for arbitrary $r$, only tri-linear combinations become $r$-linear.

All this is a direct generalization of properties of the rank $r=2$ case (rectangular complex matrix model, RCM), where the averages are bilinear in dimensions, allowed are only the diagonal bilinear products $D_{R}\left(N_{1}\right) D_{R}\left(N_{2}\right)$, and an attempt to write down an operator $\left\langle\mathcal{K}_{R_{1} R_{2}}\right\rangle \sim D_{R_{1}} D_{R_{2}}$ with $R_{1} \neq R_{2}$ fails: the operator with such a symmetry vanishes. An additional feature of RCM is that diagonal operators $\mathcal{K}_{R, R}=\chi_{R}\{P\}$, i.e. are just characters. This suggests that the tensorial operators $\chi_{R_{1}, \ldots, R_{r}}(M, \bar{M})$ which we construct in this way are direct counterparts of characters.

Among the properties that they inherit are:

(a) orthogonality;

(b) they are eigenfunctions of generalized cut-and-join operators $\hat{W}$, the tensorial counterparts of those from $[52,53]$;

(c) they form a redundant basis of the operators with non-vanishing Gaussian averages. 
The main mystery is the separation of gauge-invariant tensor model operators into two sectors. One sector consists of operators which resemble characters and possess nonvanishing Gaussian averages. It is very similar to the conventional matrix models like RCM, and should be rather straightforward to investigate. The second sector is its much bigger complement, which lies in the kernel of Gaussian averages. The quotient structure $V / W$, in the space $V$ of gauge invariant operators, where $W \neq V$ is the vector space spanned by linear combinations of operators with vanishing Gaussian averages, is a generic feature of rainbow tensor models. It is non-trivial, because $V / W$ is not a linear subspace in the linear space $V$ and can be described in different bases. The short exact sequence

$$
0 \longrightarrow W \longrightarrow V \longrightarrow V / W \longrightarrow 0
$$

implies interesting cohomological interpretations and calls for further investigation.

A possible clue to understanding this complement of $W$ is in a remarkable relation [48] which associates gauge invariant operators with Feynman diagrams in the theory of onerank-less. One of the immediate questions to address is what characterizes the subset of Feynman diagrams associated with the character sector. It would be also very useful to describe in these terms the Virasoro-like identities and, more generally, the CJ structure introduced in [12]: the two sectors should be somehow separated, and the recursion between different ranks $r$ should be lifted to the level of CJ structure. In the case of Aristotelian (rank $r=3$ ) model, there is a third description: in terms of the Grothendieck's dessins, which turns especially helpful in classifying the non-character sector in the operator space.

To conclude, in this paper we report the discovery of tensorial lifting of characters and their apparent compatibility with Gaussian averaging, which opens absolutely new perspectives to the theory of tensor models. There is a whole new world to explore, and it is now clear that it can be structured at least as well as its celebrated matrix model predecessor is. In particular, existence of this theory of tensorial characters reflects the fact that rainbow tensor models are superintegrable and exactly solvable like their well known complex matrix model "parent" [51].

\section{Acknowledgments}

We are grateful to the referee of our paper for valuable comments and an advice. A. Mironov is grateful for the hospitality of NITEP, Osaka City University as well as that of the Workshop New Trends in Integrable Systems 2019 held there during the period of September, 9-20. Our work is partly supported by JSPS KAKENHI grant Number 19K03828 and OCAMI MEXT Joint Usage/Research Center on Mathematics and Theoretical Physics (H.I.), by the grant of the Foundation for the Advancement of Theoretical Physics "BASIS" (A.Mir., A.Mor.), by RFBR grants 19-01-00680 (A.Mir.) and 19-02-00815 (A.Mor.), by joint grants 19-51-53014-GFEN-a (A.Mir., A.Mor.), 19-51-50008-YaF-a (A.Mir.), 18-5105015-Arm-a (A.Mir., A.Mor.), 18-51-45010-IND-a (A.Mir., A.Mor.). The work was also partly funded by RFBR and NSFB according to the research project 19-51-18006 (A.Mir., A.Mor.). 
Open Access. This article is distributed under the terms of the Creative Commons Attribution License (CC-BY 4.0), which permits any use, distribution and reproduction in any medium, provided the original author(s) and source are credited.

\section{References}

[1] R. Gurau et al., SIGMA. Vol. 12: Special Issue on Tensor Models, Formalism and Applications, http://www.emis.de/journals/SIGMA/Tensor_Models.html, Materials of the 2nd French-Russian Conference on Random Geometry and Physics, Paris France (2016), http://www.th.u-psud.fr/RGP16/.

[2] E. Witten, An SYK-Like Model Without Disorder, J. Phys. A 52 (2019) 474002 [arXiv: 1610.09758] [inSPIRE].

[3] R. Gurau, The complete $1 / N$ expansion of a $S Y K$-like tensor model, Nucl. Phys. B 916 (2017) 386 [arXiv:1611.04032] [INSPIRE].

[4] R. Gurau, Quenched equals annealed at leading order in the colored SYK model, EPL 119 (2017) 30003 [arXiv: 1702.04228] [INSPIRE].

[5] I.R. Klebanov and G. Tarnopolsky, Uncolored random tensors, melon diagrams and the Sachdev-Ye-Kitaev models, Phys. Rev. D 95 (2017) 046004 [arXiv:1611.08915] [InSPIRE].

[6] H. Itoyama, A. Mironov and A. Morozov, Rainbow tensor model with enhanced symmetry and extreme melonic dominance, Phys. Lett. B 771 (2017) 180 [arXiv:1703.04983] [INSPIRE].

[7] K. Bulycheva, I.R. Klebanov, A. Milekhin and G. Tarnopolsky, Spectra of Operators in Large $N$ Tensor Models, Phys. Rev. D 97 (2018) 026016 [arXiv:1707.09347] [INSPIRE].

[8] I.R. Klebanov, F. Popov and G. Tarnopolsky, TASI Lectures on Large N Tensor Models, PoS (TASI2017) 004 (2018) [arXiv: 1808.09434] [INSPIRE].

[9] P. Diaz and J.A. Rosabal, Spontaneous Symmetry Breaking in Tensor Theories, JHEP 01 (2019) 094 [arXiv: 1809.10153] [INSPIRE].

[10] F.K. Popov, Supersymmetric Tensor Model at Large $N$ and Small $\epsilon$, arXiv:1907.02440 [INSPIRE].

[11] S. Prakash and R. Sinha, Melonic Dominance in Subchromatic Sextic Tensor Models, arXiv: 1908.07178 [INSPIRE].

[12] H. Itoyama, A. Mironov and A. Morozov, Cut and join operator ring in tensor models, Nucl. Phys. B 932 (2018) 52 [arXiv:1710.10027] [INSPIRE].

[13] H. Itoyama, A. Mironov and A. Morozov, Ward identities and combinatorics of rainbow tensor models, JHEP 06 (2017) 115 [arXiv:1704.08648] [INSPIRE].

[14] A. Mironov and A. Morozov, Correlators in tensor models from character calculus, Phys. Lett. B 774 (2017) 210 [arXiv:1706.03667] [INSPIRE].

[15] R. de Mello Koch and S. Ramgoolam, From Matrix Models and Quantum Fields to Hurwitz Space and the absolute Galois Group, arXiv:1002.1634 [INSPIRE].

[16] D. Garner and S. Ramgoolam, Holographic Hierarchy in the Gaussian Matrix Model via the Fuzzy Sphere, Nucl. Phys. B 875 (2013) 244 [arXiv:1303.3246] [INSPIRE].

[17] J. Ben Geloun and S. Ramgoolam, Counting Tensor Model Observables and Branched Covers of the 2-Sphere, arXiv:1307.6490 [INSPIRE]. 
[18] R. de Mello Koch and S. Ramgoolam, Free field primaries in general dimensions: Counting and construction with rings and modules, JHEP 08 (2018) 088 [arXiv:1806.01085] [INSPIRE].

[19] P. Diaz and S.-J. Rey, Orthogonal Bases of Invariants in Tensor Models, JHEP 02 (2018) 089 [arXiv:1706.02667] [INSPIRE].

[20] R. de Mello Koch, R. Mello Koch, D. Gossman and L. Tribelhorn, Gauge Invariants, Correlators and Holography in Bosonic and Fermionic Tensor Models, JHEP 09 (2017) 011 [arXiv: 1707.01455] [INSPIRE].

[21] P. Diaz, Tensor and Matrix models: a one-night stand or a lifetime romance?, JHEP 06 (2018) 140 [arXiv:1803.04471] [INSPIRE].

[22] R.C. Avohou, J. Ben Geloun and N. Dub, On the counting of $O(N)$ tensor invariants, arXiv: 1907.04668 [INSPIRE].

[23] H. Itoyama and R. Yoshioka, Generalized cut operation associated with higher order variation in tensor models, Nucl. Phys. B 945 (2019) 114681 [arXiv: 1903.10276] [INSPIRE].

[24] A. Mironov and A. Morozov, On the complete perturbative solution of one-matrix models, Phys. Lett. B 771 (2017) 503 [arXiv:1705. 00976] [INSPIRE].

[25] R. Dijkgraaf and C. Vafa, Matrix models, topological strings and supersymmetric gauge theories, Nucl. Phys. B 644 (2002) 3 [hep-th/0206255] [InSPIRE].

[26] R. Dijkgraaf and C. Vafa, On geometry and matrix models, Nucl. Phys. B 644 (2002) 21 [hep-th/0207106] [INSPIRE].

[27] R. Dijkgraaf and C. Vafa, A Perturbative window into nonperturbative physics, hep-th/0208048 [INSPIRE].

[28] L. Chekhov and A. Mironov, Matrix models versus Seiberg-Witten/Whitham theories, Phys. Lett. B 552 (2003) 293 [hep-th/0209085] [INSPIRE].

[29] H. Itoyama and A. Morozov, The Dijkgraaf-Vafa prepotential in the context of general Seiberg-Witten theory, Nucl. Phys. B 657 (2003) 53 [hep-th/0211245] [INSPIRE].

[30] A.S. Alexandrov, A. Mironov and A. Morozov, Unified description of correlators in non-Gaussian phases of Hermitean matrix model, Int. J. Mod. Phys. A 21 (2006) 2481 [hep-th/0412099] [INSPIRE].

[31] A.S. Alexandrov, A. Mironov and A. Morozov, Solving Virasoro constraints in matrix models, Fortsch. Phys. 53 (2005) 512 [hep-th/0412205] [INSPIRE].

[32] L. Chekhov, A. Marshakov, A. Mironov and D. Vasiliev, DV and WDVV, Phys. Lett. B 562 (2003) 323 [hep-th/0301071] [INSPIRE].

[33] L. Chekhov, A. Marshakov, A. Mironov and D. Vasiliev, Complex geometry of matrix models, Proc. Steklov Inst. Math. 251 (2005) 254 [hep-th/0506075] [INSPIRE].

[34] A. Mironov, Matrix models vs. matrix integrals, Theor. Math. Phys. 146 (2006) 63 [hep-th/0506158] [INSPIRE].

[35] A. Mironov, A. Morozov and Z. Zakirova, Comment on integrability in Dijkgraaf-Vafa beta-ensembles, Phys. Lett. B 711 (2012) 332 [arXiv:1202.6029] [INSPIRE].

[36] A. Mironov and A. Morozov, Sum rules for characters from character-preservation property of matrix models, JHEP 08 (2018) 163 [arXiv:1807.02409] [INSPIRE]. 
[37] A. Morozov, A. Popolitov and S. Shakirov, On $(q, t)$-deformation of Gaussian matrix model, Phys. Lett. B $\mathbf{7 8 4}$ (2018) 342 [arXiv: 1803.11401] [InSPIRE].

[38] R. Lodin, A. Popolitov, S. Shakirov and M. Zabzine, Solving q-Virasoro constraints, arXiv: 1810.00761 [INSPIRE].

[39] C. Cordova, B. Heidenreich, A. Popolitov and S. Shakirov, Orbifolds and Exact Solutions of Strongly-Coupled Matrix Models, Commun. Math. Phys. 361 (2018) 1235 [arXiv: 1611.03142] [INSPIRE].

[40] T. Morris, Checkered surfaces and complex matrices, Nucl. Phys. B 356 (1991) 703.

[41] Yu. Makeenko, Complex-matrix model and 2D quantum gravity, Pis'ma Zh. Eksp. Teor. Fiz. $\mathbf{5 2}(1990) 885$.

[42] Yu. Makeenko, A. Marshakov, A. Mironov and A. Morozov, Continuum versus discrete Virasoro in one matrix models, Nucl. Phys. B 356 (1991) 574 [InSPIRE].

[43] H. Itoyama, A. Mironov and A. Morozov, From Kronecker to tableau pseudo-characters in tensor models, Phys. Lett. B 788 (2019) 76 [arXiv: 1808.07783] [INSPIRE].

[44] J. Ben Geloun and S. Ramgoolam, Tensor Models, Kronecker coefficients and Permutation Centralizer Algebras, JHEP 11 (2017) 092 [arXiv: 1708. 03524] [INSPIRE].

[45] D.E. Littlewood, The theory of group characters and matrix representations of groups, Oxford University Press, Oxford U.K. (1958).

[46] M. Hammermesh, Group Theory and Its Application to Physical Problems, Dover Books on Physics, Dover Publications, Mineola U.S.A. (1989).

[47] G.D. James and A. Kerber, The Representation Theory of the Symmetric Group, Encyclopedia of Mathematics and its Applications, Cambridge University Press, Cambridge U.K. (2009).

[48] N. Amburg, H. Itoyama, A. Mironov, A. Morozov, D. Vasiliev and R. Yoshioka, Correspondence between Feynman diagrams and operators in quantum field theory that emerges from tensor model, arXiv:1911.10574 [INSPIRE].

[49] I.G. Macdonald, Symmetric functions and Hall polynomials, second edition, Oxford University Press, Oxford U.K. (1995).

[50] A. Morozov, Cauchy formula and the character ring, Eur. Phys. J. C 79 (2019) 76 [arXiv: 1812.03853] [INSPIRE].

[51] H. Itoyama, A. Mironov and A. Morozov, Complete solution to Gaussian tensor model and its integrable properties, arXiv:1910.03261 [INSPIRE].

[52] A. Mironov, A. Morozov and S. Natanzon, Complete Set of Cut-and-Join Operators in Hurwitz-Kontsevich Theory, Theor. Math. Phys. 166 (2011) 1 [arXiv:0904.4227] [INSPIRE].

[53] A. Mironov, A. Morozov and S. Natanzon, Algebra of differential operators associated with Young diagrams, J. Geom. Phys. 62 (2012) 148 [arXiv:1012.0433] [InSPIRE].

[54] D. Goulden, D.M. Jackson and A. Vainshtein, The number of ramifified coverings of the sphere by torus and surfaces of higher genera, Ann. Comb. 4 (2000) 27 [math/9902125].

[55] S. Kharchev, A. Marshakov, A. Mironov and A. Morozov, Generalized Kazakov-Migdal-Kontsevich model: Group theory aspects, Int. J. Mod. Phys. A 10 (1995) 2015 [hep-th/9312210] [InSPIRE]. 
[56] A. Orlov and D.M. Shcherbin, Hypergeometric solutions of soliton equations, Theor. Math. Phys. 128 (2001) 906.

[57] A. Orlov, Hypergeometric functions as infinite-soliton Tau functions, Theor. Math. Phys. 146 (2006) 183.

[58] A. Alexandrov, A. Mironov, A. Morozov and S. Natanzon, Integrability of Hurwitz Partition Functions. I. Summary, J. Phys. A 45 (2012) 045209 [arXiv: 1103.4100] [InSPIRE].

[59] A. Alexandrov, A. Mironov, A. Morozov and S. Natanzon, On KP-integrable Hurwitz functions, JHEP 11 (2014) 080 [arXiv: 1405.1395] [INSPIRE]. 